JOURNAL OF THE

AMERICAN MATHEMATICAL SOCIETY

Volume 12, Number 2, April 1999, Pages 305-333

S 0894-0347(99)00291-X

\title{
A CONVERSE TO A THEOREM OF ADAMYAN, AROV AND KREIN
}

\author{
J. AGLER AND N. J. YOUNG
}

\section{INTRODUCTION}

A classical theorem of Pick gives a criterion for interpolation by analytic functions in the open unit disc $\mathbb{D}$ subject to an $H^{\infty}$-norm bound. This result has substantial generalizations in two different directions. On the one hand, one can replace $H^{\infty}$ by the multiplier algebras of certain Hilbert function spaces, some of them having no connection with analyticity $[\mathrm{Ag} 1, \mathrm{Ag} 2]$. On the other hand, one can obtain a criterion for interpolation by meromorphic functions with a prescribed number of poles in $\mathbb{D}$ and with an $L^{\infty}$-norm bound on the unit circle $\mathbb{T}$; this is a classical result of Akhiezer [Ak], now better known in the form of the far-reaching generalizations due to Adamyan, Arov and Krein [AAK]. In each case the criterion is in terms of the signature of a "Pick matrix" constructed from the interpolation data and the reproducing kernel of the appropriate Hilbert function space (i.e. $H^{2}$ in the case of the AAK theorem). It is therefore conceivable that there might be a common generalization which would hold for a significant class of function spaces. After all, the analogue of Pick's theorem is true for the Dirichlet space $D$ of analytic functions in $\mathbb{D}$ with finite Dirichlet integral $[\mathrm{Ag} 1]$ and for the space $W^{1,2}[a, b]$ of $L^{2}$ functions $f$ on $[a, b]$ for which $f^{\prime} \in L^{2}(a, b)[\mathrm{Ag} 2]$. Might not an analogue of the Akhiezer-Adamyan-Arov-Krein theorem hold for these spaces? This natural question was posed in [Q2].

Pick's theorem has long held the attention of analysts as one of the most elegant of all interpolation results. However, there are grounds beyond the aesthetic ones for its continued prominence. Knowledge that the analogue of Pick's theorem holds for a particular Hilbert function space gives a powerful tool for the conversion of $L^{\infty}$-type problems to Hilbert space problems; this principle is brought out in [MS], where among other things it is used to give a relatively simple proof of Carleson's theorem on interpolation sequences for $H^{\infty}$ and also to characterize geometrically the interpolation sequences for multipliers of the Dirichlet space. Since about 1980 the Pick property has played an important role in linear control theory. The feedback controllers which internally stabilize a given linear system can be described by analytic functions satisfying a finite set of interpolation conditions, so

Received by the editors May 28, 1997.

1991 Mathematics Subject Classification. Primary 46E22; Secondary 47B38.

Key words and phrases. Interpolation, reproducing kernel, multiplier, Pick's theorem, Adamyan-Arov-Krein theorem, Akhiezer's theorem.

J. Agler's research was supported by an NSF grant in Modern Analysis.

(C)1999 American Mathematical Society 
that constructing a stabilizing controller with gain bounded by 1 is a Pick interpolation problem [DFT]. Applications of the Pick property to circuit theory can be found in [DGK, H]. Akhiezer's generalization of Pick's theorem (usually in the Adamyan-Arov-Krein version) has led to an effective approach to model reduction [G].

Pick's and Akhiezer's theorems have also served as test problems for techniques in function theory and operator theory. The applications call for far-reaching refinements of the classical theorems, and numerous approaches have been developed to proving generalizations. Closely related approaches of Sarason [S] and Adamyan, Arov and Krein [AAK] prove them using compressions of the shift operator and Hankel operations on $H^{2}$ respectively. Other powerful techniques are the commutant lifting theory $[\mathrm{FF}]$, the invariant subspace theory for indefinite Hardy spaces $[\mathrm{BH}]$ and at least four other radically different methods. A recent development along novel lines is a series of generalizations to functions of severable variables by means of a general lifting theorem by Cotlar and Sadosky [CS].

The approaches we have listed are all closely tied to the Hardy space $H^{2}$ and to the Szegö kernel. Investigation of the Pick property for other Hilbert spaces of functions in [Ag1, Ag2] had to proceed by a more "bare hands" method, involving a point-by-point construction of interpolating functions. So it is with the present paper, where we study the extension of Akhiezer's theorem to other function spaces. The original theorem of Akhiezer is couched in terms of meromorphic functions. Here we study functions on an arbitrary set, possibly without differentiable structure, and so we need a reformulation of Akhiezer's theorem which has an analogue in the general setting. Let $H$ be a Hilbert space of functions on a set $\Omega$ and let $k: \Omega \times \Omega \rightarrow \mathbb{C}$ be the reproducing kernel of $H$. Consider distinct points $\lambda_{1}, \ldots, \lambda_{n} \in \Omega$ and complex numbers $z_{1}, \ldots, z_{n}$; we regard these as data for an interpolation problem. The Pick matrix corresponding to these data is the $n \times n$ matrix $\left[\left(1-z_{i} \bar{z}_{j}\right) k\left(\lambda_{i}, \lambda_{j}\right)\right]_{i, j=1}^{n}$. One says [Q1] that Pick's theorem holds for $(H, \Omega)$ if the following two statements are equivalent for any set of interpolation data:

(1) there exists a function $\varphi: \Omega \rightarrow \mathbb{C}$ which is a contractive multiplier of $H$ and satisfies $\varphi\left(\lambda_{i}\right)=z_{i}, 1 \leq i \leq n$;

(2) the corresponding Pick matrix is positive semidefinite.

The classical result $[\mathrm{P}]$ is usually stated as a criterion for the existence of an analytic interpolating function bounded by one in modulus, but it can also be formulated as the statement that Pick's theorem holds for $H^{2}$. The results of [Ag1, Ag2] are that Pick's theorem holds for $D$ and for $W^{1,2}[a, b]$. Similarly we can formulate the Akhiezer-Adamyan-Arov-Krein generalization of Pick's theorem by saying that when $H$ is $H^{2}$ and $\Omega$ is $\mathbb{D}$, for any finite set of interpolation data and any non-negative integer $m$, the following two statements are equivalent:

(1) there exists a function $\varphi$ defined at all but at most $m$ points of $\Omega$ which contractively multiplies an $m$-codimensional closed subspace of $H$ into $H$ and satisfies $\varphi\left(\lambda_{i}\right)=z_{i}, 1 \leq i \leq n$

(2) the corresponding Pick matrix has at most $m$ negative eigenvalues.

For $H=H^{2}$, condition (1) amounts to saying there is an interpolating meromorphic function with at most $m$ poles bounded by 1 in modulus on $\mathbb{T}$ (actually a precise statement is slightly more cumbersome since account must be taken of certain singular cases), which is the formulation given by Akhiezer. Accordingly we say that $(H, \Omega)$ has the $m$-AAK property if (1) is equivalent to (2) for any finite set of interpolation data (a more precise definition is given below). The question 
we address is:

Which Hilbert function spaces have the $m$-AAK property for all non-negative integers $m$ ?

Our main result, Theorem 3.12, answers this question. It constitutes a converse to Akhiezer's result that $H^{2}$ has the $m$-AAK property for all $m$ : it asserts that, subject to modest hypotheses on $H$, if $H$ has the 0 -AAK property and the 1 -AAK property, then the reproducing kernel of $H$ is a Szegö kernel - roughly speaking, $(H, \Omega)$ is a subspace of $H^{2}$ up to rescaling and a change of variable. It follows that $D$ and $W^{1,2}[a, b]$ are examples of spaces having the 0 -AAK property but not the 1 -AAK property. The restriction of the Bergman space of the disc, $L_{a}^{2}(\mathbb{D})$, to any 3 points is an example of a space having the 1-AAK property but not the 0-AAK property (actually this is a slight over-simplification: see Corollary 3.4 below). In the course of establishing Theorem 3.12 we prove our other main result, Theorem 1.8 , which characterizes the $m$-AAK property for spaces of functions on infinite sets in terms of the restrictions of the kernel to finite sets. This result contains the Akhiezer-Adamyan-Arov-Krein theorem. It enables us to concentrate on the restrictions of the kernel to small sets; for example, it turns out that the signs of the determinants of the (entrywise) reciprocals of the $3 \times 3$ principal submatrices of the kernel play a key role (Theorem 3.1).

\section{DEFINITIONS AND NOTATION}

By a Hilbert function space we mean a pair $(H, \Omega)$ where $\Omega$ is a set and $H$ is a Hilbert space of functions on $\Omega$ such that evaluation at any point of $\Omega$ is a continuous linear functional on $H$. When there is no risk of confusion we may sometimes write $H$ in place of $(H, \Omega)$; however we shall often need to be precise about the domain under consideration. Thus, if $\Omega^{\prime} \nsubseteq \subseteq \Omega,\left(H \mid \Omega^{\prime}, \Omega^{\prime}\right)$ is a different Hilbert function space from $(H, \Omega)$, where the vertical bar denotes restriction and $H \mid \Omega^{\prime} \stackrel{\text { def }}{=}\left\{f \mid \Omega^{\prime}: f \in H\right\}$. Nevertheless the natural mapping $f \mapsto f \mid \Omega^{\prime}$ may be a unitary mapping between the Hilbert spaces $H$ and $H \mid \Omega^{\prime}$. Indeed, this will be so if and only if $\Omega^{\prime}$ is a set of uniqueness for $H$, meaning that if $f, g \in H$ and $f\left|\Omega^{\prime}=g\right| \Omega^{\prime}$, then $f=g$. A Hilbert function space $(H, \Omega)$ has a reproducing kernel, that is, a function $k: \Omega \times \Omega \rightarrow \mathbb{C}$ with defining property

$$
\left\langle f, k_{\lambda}\right\rangle=f(\lambda) \quad \text { for all } \quad f \in H \quad \text { and } \quad \lambda \in \Omega
$$

where $k_{\lambda}=k(\cdot, \lambda) \in H$. We say that $k$ is a non-singular kernel if the vectors $k_{\lambda}, \lambda \in \Omega$, are linearly independent.

Now consider a fixed Hilbert space $H$ of functions on a set $\Omega$ with non-singular kernel $k$. For $\psi: D_{\psi} \subset \Omega \rightarrow \mathbb{C}$ and $h \in H$ we denote by $\psi h$ the pointwise product of $\psi$ and $h \mid D_{\psi}$, so that $\psi h$ is a function on $D_{\psi}$. For $E \subset H$ we denote by $\psi E$ the set $\{\psi h: h \in E\}$, so that $\psi E$ is a set of functions on $D_{\psi}$. If $E$ is a closed linear subspace of $H$, if $D_{\psi}$ is a set of uniqueness and $\psi E \subset H \mid D_{\psi}$ we define the linear operator $M_{\psi, E}: E \rightarrow H$ by $M_{\psi, E} h=g$ where $g$ is the unique element of $H$ which extends $\psi h$. By the closed graph theorem $M_{\psi, E}$ is continuous. If $D_{\psi}$ is a set of uniqueness of $H$ we shall say that $\alpha \in \Omega$ is a pseudopole of $\psi$ (relative to $H$ ) if there exists $h \in H$ such that $h(\alpha)=0$ and $\psi h$ has an extension to a function $g \in H$ (necessarily unique) such that $g(\alpha) \neq 0$. Of course this can only happen if $\alpha \notin D_{\psi}$.

Definition 0.1. Let $\psi$ be a complex-valued function on a subset $D_{\psi}$ of $\Omega$ and let $m \in \mathbb{Z}^{+}$, the set of non-negative integers. We say that $\psi$ is an m-pseudomultiplier 
of $H$ on $\Omega$ if

(1) $D_{\psi}$ is a set of uniqueness for $H$;

(2) $\Omega \backslash D_{\psi}$ consists of pseudopoles of $\psi$ and contains at most $m$ points;

(3) there is a closed subspace $E$ of codimension $m$ in $H$ such that $\psi E \subset H \mid D_{\psi}$.

For an $m$-pseudomultiplier $\psi$ of $H$ we define $s_{m}(\psi)$ to be the infimum of $\left\|M_{\psi, E}\right\|$ over all closed $m$-codimensional subspaces $E$ of $H$ such that $\psi E \subset H \mid D_{\psi}$. We say that $\psi$ is a pseudomultiplier of $H$ if it is an $m$-pseudomultiplier of $H$ for some non-negative integer $m$.

There are two standard examples of 1-pseudomultipliers to keep in mind, both on $\left(H^{2}, \mathbb{D}\right): \varphi_{1}(z)=1 / z$ and $\varphi_{2}=\chi_{\{0\}}$, the characteristic or indicator function of the singleton set $\{0\}$. Note that $s_{1}\left(\varphi_{1}\right)=1$ and $s_{1}\left(\varphi_{2}\right)=0$. It might appear that the assumptions about $D_{\varphi}$ in Definition 0.1 are unduly strong, but the main result of $[\mathrm{AY}]$, Theorem 2.1, shows that weaker assumptions lead to essentially the same objects, subject only to the mild hypotheses that $D_{\varphi}$ be a set of uniqueness for the class $H H \stackrel{\text { def }}{=}\{f g: f, g \in H\}$ and that $k$ be non-singular.

Definition 0.2. Let $(H, \Omega)$ be a Hilbert function space with reproducing kernel $k$ and let $m$ be a non-negative integer. We say that $(H, \Omega)$ has the $m$-AAK property if the following two conditions are equivalent for any choice of $n \in \mathbb{N}$, distinct points $\lambda_{1}, \ldots, \lambda_{n} \in \Omega$ and arbitrary complex numbers $z_{1}, \ldots, z_{n}$ :

(1) there exists an $m$-pseudomultiplier $\varphi$ of $(H, \Omega)$ such that $s_{m}(\varphi) \leq 1$ and $\varphi\left(\lambda_{j}\right)=z_{j}, 1 \leq j \leq n$

(2) the matrix

$$
\left[\left(1-z_{i} \bar{z}_{j}\right) k\left(\lambda_{i}, \lambda_{j}\right)\right]_{i, j=1}^{n}
$$

has at most $m$ negative eigenvalues, counting multiplicities.

Our goal is to characterize Hilbert function spaces with the 0-AAK property and the 1-AAK property. The conclusion is in terms of Szegö kernels. We say that a kernel $k: \Omega \times \Omega \rightarrow \mathbb{C}$ is a Szegö kernel if there exist functions $a: \Omega \rightarrow \mathbb{C} \backslash\{0\}$ and $b: \Omega \rightarrow \mathbb{D}$ such that $b$ is injective and

$$
k(\lambda, \mu)=\frac{a(\lambda) \bar{a}(\mu)}{1-b(\lambda) \bar{b}(\mu)} .
$$

\section{OnE POINT EXTENSIONS}

In this section we characterize the $m$-AAK property in terms of operators on finite-dimensional spaces. Our approach is based on constructing pseudomultipliers one point at a time. It is a little surprising that, say, a meromorphic function can successfully be constructed in this finitary, point-by-point manner, but study of the case $m=0$ (the Pick property) shows that it is indeed possible [Ag1, Ag2]. One thinks in terms of associated multiplication operators and keeps control of the operator norm. When $m>0$ this technique has to be modified since multiplication by a pseudomultiplier is no longer a bounded operator on $H$. Instead of the operator norm we use the $m$ th $s$-number or singular value of an operator, and we are faced with the problem of extending operators with preservation of the $m$ th $s$-number. This is a more subtle problem than the corresponding one for the operator norm [CG], and it cannot always be achieved exactly.

Throughout this section we consider a fixed Hilbert function space $(H, \Omega)$ with non-singular reproducing kernel $k$. We first wish to derive a necessary condition for 
$H$ to have the $m$-AAK property. The idea is simplest in the case $m=0$. Suppose that $H$ has the 0-AAK (or Pick) property. That is, for any choice of distinct points $\lambda_{1}, \ldots, \lambda_{n} \in \Omega$ and any $z_{1}, \ldots, z_{n} \in \mathbb{C}$ such that

$$
\left[\left(1-z_{i} \bar{z}_{j}\right) k\left(\lambda_{i}, \lambda_{j}\right)\right]_{i, j=1}^{n} \geq 0
$$

there exists a contractive multiplier $\varphi$ of $H$ such that $\varphi\left(\lambda_{i}\right)=z_{i}, 1 \leq i \leq n$. There is a standard operator-theoretic way of viewing the Pick condition (1.1). Introduce the space

$$
\mathcal{M} \stackrel{\text { def }}{=} \operatorname{span}\left\{k_{\lambda_{1}}, \ldots, k_{\lambda_{n}}\right\} \subset H
$$

and the linear operator $T$ on $\mathcal{M}$ given by

$$
T k_{\lambda_{j}}=\bar{z}_{j} k_{\lambda_{j}}, \quad 1 \leq j \leq n .
$$

The matrix of $1-T^{*} T$ with respect to the basis $k_{\lambda_{1}}, \ldots, k_{\lambda_{n}}$ is precisely the Pick matrix, i.e. the left hand side of (1.1), and so the condition (1.1) is equivalent to $\|T\| \leq 1$. The Pick property of $H$ thus tells us that if the data $\lambda_{j}, z_{j}$ are such that $\|T\| \leq 1$, then there is an interpolating multiplier $\varphi$ such that $\left\|M_{\varphi}\right\| \leq 1$, where $M_{\varphi} \in \mathcal{L}(H)$ is the operation of multiplication by $\varphi$. It is readily seen, in this case, that $T$ is the restriction of $M_{\varphi}^{*}$ to its invariant subspace $\mathcal{M}$, so that we can regard the Pick property as an extension property for certain operators on finite-dimensional subspaces of $H$. This viewpoint leads to a necessary condition for $(H, \Omega)$ to have the Pick property. Let $T, \mathcal{M}$ and $\varphi$ be as above. Choose any $\alpha \in \Omega \backslash\left\{\lambda_{1}, \ldots, \lambda_{n}\right\}$ and let

$$
\tilde{\mathcal{M}}=\operatorname{span}\left\{k_{\lambda_{1}}, \ldots, k_{\lambda_{n}}, k_{\alpha}\right\} \cap k_{\alpha}^{\perp} .
$$

Then $\tilde{\mathcal{M}}$ is an $n$-dimensional subspace of $H$ and a basis of $\tilde{\mathcal{M}}$ is $\tilde{k}_{\lambda_{1}}, \ldots, \tilde{k}_{\lambda_{n}}$ where

$$
\tilde{k}_{\lambda_{j}}=P_{\tilde{\mathcal{M}}} k_{\lambda_{j}}=k_{\lambda_{j}}-\frac{k\left(\alpha, \lambda_{j}\right)}{k(\alpha, \alpha)} k_{\alpha}
$$

Let $\tilde{T}$ be the compression of $M_{\varphi}^{*}$ to $\tilde{\mathcal{M}}$. Then

$$
\begin{aligned}
\tilde{T} \tilde{k}_{\lambda_{j}} & =P_{\tilde{\mathcal{M}}} M_{\varphi}^{*} \tilde{k}_{\lambda_{j}}=P_{\tilde{\mathcal{M}}}\left(\bar{\varphi}\left(\lambda_{j}\right) k_{\lambda_{j}}-\frac{k\left(\alpha, \lambda_{j}\right)}{k(\alpha, \alpha)} \bar{\varphi}(\alpha) k_{\alpha}\right) \\
& =\bar{z}_{j} \tilde{k}_{\lambda_{j}}
\end{aligned}
$$

since $P_{\tilde{\mathcal{M}}} k_{\alpha}=0$. Now the $n$-dimensional operator $\tilde{T}$ is determined by (1.5), which expresses $\tilde{T}$ entirely in terms of the data and the chosen point $\alpha$. On the other hand, since $\tilde{T}$ is a compression of the contraction $M_{\varphi}^{*}$, we must have $\|\tilde{T}\| \leq 1$. We have shown that the following is a necessary condition that $(H, \Omega)$ (or equivalently, the kernel $k$ ) have the Pick property:

For any choice of distinct points $\lambda_{1}, \ldots, \lambda_{n}, \alpha \in \Omega$ and of $z_{1}, \ldots, z_{n} \in \mathbb{C}$, the operators $T, \tilde{T}$ given by (1.3) and (1.5) respectively satisfy $\|\tilde{T}\| \leq\|T\|$.

We shall now show that, subject to a mild hypothesis, the expected variant is valid with "Pick" replaced by " $m$-AAK" and $\|\cdot\|$ replaced by $s_{m}$.

Lemma 1.1. Suppose that $D_{\varphi} \subset \Omega$ is a set of uniqueness for $H$ and for $H H$. If $\varphi: D_{\varphi} \subset \Omega \rightarrow \mathbb{C}$ is a pseudomultiplier of $H$ and $\alpha \in \Omega \backslash D_{\varphi}$, then any $h \in H$ such that $\varphi h \in H \mid D_{\varphi}$ must vanish at $\alpha$. 
Proof. By the definition of pseudomultiplier, $\alpha$ is a pseudopole of $\varphi$. That is, there exist $u, g \in H$ such that $u(\alpha)=0, \varphi u=g \mid D_{\varphi}$ and $g(\alpha) \neq 0$. Suppose $\varphi h=f \mid D_{\varphi}$ where $f \in H$. On $D_{\varphi}$ we have

$$
g h-u f=\varphi u h-u \varphi h=0 .
$$

Thus $g h$ and $u f$ agree on $D_{\varphi}$, which by hypothesis is a set of uniqueness for $H H$. Thus $g h=u f$. Since $u(\alpha)=0$ and $g(\alpha) \neq 0$, we have $h(\alpha)=0$.

Remark. The conclusion would not follow if the hypothesis on $H H$ were removed. [AY, Example 2.2] exhibits a 1-pseudomultiplier $\varphi$ and $\alpha \in \Omega \backslash D_{\varphi}$ such that $\varphi h \in H \mid D_{\varphi}$ for all $h \in H$, while not all $h$ vanish at $\alpha$.

Lemma 1.2. Suppose every cofinite set of uniqueness for $H$ in $\Omega$ is also a set of uniqueness for $H H$. If $m$ is a non-negative integer, $\lambda_{1}, \ldots, \lambda_{n}, \alpha$ are distinct points of $\Omega, z_{1}, \ldots, z_{n} \in \mathbb{C}$ and $\varphi$ is an m-pseudomultiplier of $H$ such that $\varphi\left(\lambda_{j}\right)=z_{j}, 1 \leq$ $j \leq n$, then the operator $\tilde{T}$ defined by (1.5) satisfies

$$
s_{m}(\tilde{T}) \leq s_{m}(\varphi)
$$

Proof. Let $\varepsilon>0$. By hypothesis there exists an $m$-codimensional subspace $E$ of $H$ such that $\varphi E \subset H \mid D_{\varphi}$ and the operator $X_{\varphi}: E \rightarrow H$ given by

$$
\left(X_{\varphi} f\right) \mid D_{\varphi}=\varphi f, \quad f \in E,
$$

satisfies

$$
\left\|X_{\varphi}\right\|<s(\varphi)+\varepsilon
$$

Observe that $X_{\varphi}^{*}: H \rightarrow E$ satisfies, for any $\lambda \in D_{\varphi}$,

$$
X_{\varphi}^{*} k_{\lambda}=\bar{\varphi}(\lambda) P_{E} k_{\lambda}
$$

Hence, for $1 \leq j \leq n$ there exists $u_{j} \in E^{\perp}$ such that

$$
\begin{aligned}
X_{\varphi}^{*} k_{\lambda_{j}} & =\bar{\varphi}\left(\lambda_{j}\right) k_{\lambda_{j}}+u_{j} \\
& =\bar{z}_{j} k_{\lambda_{j}}+u_{j} .
\end{aligned}
$$

Let $T_{1}=P_{\tilde{\mathcal{M}}} X_{\varphi}^{*} \mid \tilde{\mathcal{M}}: \tilde{\mathcal{M}} \rightarrow \tilde{\mathcal{M}}$. For $1 \leq j \leq n$ and some $c_{j} \in \mathbb{C}$,

$$
\begin{aligned}
T_{1} \tilde{k}_{\lambda_{j}} & =P_{\tilde{\mathcal{M}}} X_{\varphi}^{*}\left(k_{\lambda_{j}}-c_{j} k_{\alpha}\right) \\
& =P_{\tilde{\mathcal{M}}}\left(\bar{z}_{j} k_{\lambda_{j}}+u_{j}-c_{j} X_{\varphi}^{*} k_{\alpha}\right) \\
& =\bar{z}_{j} \tilde{k}_{\lambda_{j}}+P_{\tilde{\mathcal{M}}} u_{j}-c_{j} P_{\tilde{\mathcal{M}}} X_{\varphi}^{*} k_{\alpha} \\
& =\tilde{T} \tilde{k}_{\lambda_{j}}+P_{\tilde{\mathcal{M}}} u_{j}-c_{j} P_{\tilde{\mathcal{M}}} X_{\varphi}^{*} k_{\alpha} .
\end{aligned}
$$

Thus

$$
\operatorname{Range}\left(T_{1}-\tilde{T}\right) \subset \operatorname{span}\left\{P_{\tilde{\mathcal{M}}} E^{\perp}, P_{\tilde{\mathcal{M}}} X_{\varphi}^{*} k_{\alpha}\right\}
$$

We claim that the right hand side has dimension at most $m$. For suppose $\alpha \in D_{\varphi}$. Then for some $u \in E^{\perp}$

$$
X_{\varphi}^{*} k_{\alpha}=\bar{\varphi}(\alpha) k_{\alpha}+u
$$

and so, since $P_{\tilde{\mathcal{M}}} k_{\alpha}=0$,

$$
P_{\tilde{\mathcal{M}}} X_{\varphi}^{*} k_{\alpha}=P_{\tilde{\mathcal{M}}} u \in P_{\tilde{\mathcal{M}}} E^{\perp}
$$

Hence

$$
\operatorname{Range}\left(T_{1}-\tilde{T}\right) \subset P_{\tilde{\mathcal{M}}} E^{\perp}
$$


which has dimension $\leq m$. Alternatively $\alpha \notin D_{\varphi}$. In that case, by Lemma 1.1, every element of $E$ vanishes at $\alpha$, i.e. $E \subset k_{\alpha}^{\perp}$. Hence $k_{\alpha} \in E^{\perp}$, and since $P_{\tilde{\mathcal{M}}} k_{\alpha}=0$ it follows that

$$
\operatorname{dim} P_{\mathcal{M}} E^{\perp} \leq \operatorname{dim} E^{\perp}-1 \leq m-1,
$$

and so

$$
\operatorname{dim} \operatorname{span}\left\{P_{\tilde{\mathcal{M}}} E^{\perp}, P_{\tilde{\mathcal{M}}} X_{\varphi}^{*} k_{\alpha}\right\} \leq m .
$$

In either case $T_{1}-\tilde{T}$ has rank at most $m$. Since $s_{m}(\tilde{T})$ is the distance of $\tilde{T}$ from the set of operators of rank at most $m$ we have

$$
\begin{aligned}
s_{m}(\tilde{T}) & \leq\left\|\tilde{T}-\left(\tilde{T}-T_{1}\right)\right\|=\left\|T_{1}\right\| \\
& \leq\left\|X_{\varphi}^{*}\right\|<s_{m}(\varphi)+\varepsilon .
\end{aligned}
$$

Since $\varepsilon$ was arbitrary we have

$$
s_{m}(\tilde{T}) \leq s_{m}(\varphi)
$$

Corollary 1.3. Suppose that $(H, \Omega)$ has the $m$-AAK property and that every cofinite set of uniqueness for $H$ in $\Omega$ is also a set of uniqueness for $H H$. For any choice of distinct points $\lambda_{1}, \ldots, \lambda_{n}, \alpha \in \Omega$ and of $z_{1}, \ldots, z_{n} \in \mathbb{C}$, the operators $T$ and $\tilde{T}$ given by (1.3) and (1.5) respectively satisfy

$$
s_{m}(\tilde{T}) \leq s_{m}(T) .
$$

Proof. Suppose $\lambda_{j}, z_{j}$ are such that $s_{m}(T) \leq 1$. Then $1-T^{*} T$ has at most $m$ negative eigenvalues. Expressing $1-T^{*} T$ as a matrix with respect to $k_{\lambda_{1}}, \ldots, k_{\lambda_{n}}$ we find that the Pick matrix $\left[\left(1-z_{i} \bar{z}_{j}\right) k\left(\lambda_{i}, \lambda_{j}\right)\right]_{i, j=1}^{n}$ has at most $m$ negative eigenvalues. By the $m$-AAK property, there is an $m$-pseudomultiplier $\varphi$ of $H$ such that $\varphi\left(\lambda_{j}\right)=z_{j}$ and $s_{m}(\varphi) \leq 1$. By Lemma 1.2 it follows that $s_{m}(\tilde{T}) \leq 1$. Hence

$$
s_{m}(\tilde{T}) \leq s_{m}(T) .
$$

For any operator $A$ we denote $\left(A^{*} A\right)^{1 / 2}$ by $|A|$.

Corollary 1.4. Suppose that $(H, \Omega)$ has the $m$-AAK property for every $m$ and that every cofinite set of uniqueness for $H$ in $\Omega$ is also a set of uniqueness for $H H$. Then, for any choice of data $\lambda_{1}, \ldots, \lambda_{n} \in \Omega, z_{1}, \ldots, z_{n} \in \mathbb{C}$, the operators $T, \tilde{T}$ given by (1.3), (1.5) respectively satisfy

$$
|T| \cong|\tilde{T}| .
$$

Here $\cong$ denotes unitary equivalence.

Proof. By Corollary 1.3 we have $s_{m}(\tilde{T}) \leq s_{m}(T)$ for all $m$. Furthermore

$$
s_{0}(T) s_{1}(T) \ldots s_{n-1}(T)=|\operatorname{det} T|=\left|z_{1} z_{2} \ldots z_{n}\right|
$$

and likewise $s_{0}(\tilde{T}) s_{1}(\tilde{T}) \ldots s_{n-1}(\tilde{T})=\left|z_{1} z_{2} \ldots z_{n}\right|$. Provided that all $z_{j}$ are nonzero it follows that $s_{j}(T)=s_{j}(\tilde{T}), 1 \leq j \leq n$, and hence that $|T| \cong|\tilde{T}|$. To see that the conclusion remains true even if some $z_{j}$ are zero we appeal to a theorem of Specht (see for example [K, Theorem 63]), according to which two operators $A, B$ on $n$-dimensional Hilbert spaces are unitarily equivalent if and only if

$$
\operatorname{tr} f\left(A, A^{*}\right)=\operatorname{tr} f\left(B, B^{*}\right)
$$


for every monomial $f$ in a pair of non-commuting variables. It is clear that $\operatorname{tr} f\left(T, T^{*}\right)$ and $\operatorname{tr} f\left(\tilde{T}, \tilde{T}^{*}\right)$ vary continuously as functions of $z_{1}, \ldots, z_{n}$. Since they agree when the $z_{j}$ are all non-zero they must agree for all $z_{j}$. Thus $|T| \cong|\tilde{T}|$.

Let us introduce some terminology for the conditions arising in Corollary 1.3.

Definition 1.5. Let $(H, \Omega)$ be a Hilbert function space with kernel $k$ and let $m$ be a non-negative integer. We say that $H$ has the compression property for $s_{m}$ if, for any $n \in \mathbb{N}$ and any choice of distinct points $\lambda_{1}, \ldots, \lambda_{n}, \alpha \in \Omega$ and of $z_{1}, \ldots, z_{n} \in \mathbb{C}$, the operators $T, \tilde{T}$ on

$$
\mathcal{M} \stackrel{\text { def }}{=} \operatorname{span}\left\{k_{\lambda_{1}}, \ldots, k_{\lambda_{n}}\right\}, \quad \tilde{\mathcal{M}} \stackrel{\text { def }}{=} \operatorname{span}\left\{\mathcal{M}, k_{\alpha}\right\} \cap k_{\alpha}^{\perp}
$$

given by

$$
T k_{\lambda_{j}}=\bar{z}_{j} k_{\lambda_{j}}, \quad \tilde{T} P_{\tilde{\mathcal{M}}} k_{\lambda_{j}}=\bar{z}_{j} P_{\tilde{\mathcal{M}}} k_{\lambda_{j}}, \quad 1 \leq j \leq n,
$$

respectively, satisfy

$$
s_{m}(\tilde{T}) \leq s_{m}(T) .
$$

It will often be enough to assume that the relation $s_{m}(\tilde{T}) \leq s_{m}(T)$ holds for the particular values $n=2$ and $n=3$; in these cases we shall say that $H$ has the compression property for $s_{m}$ for pairs of points or for triples of points respectively.

Thus Corollary 1.3 can be expressed as follows. Suppose every cofinite set of uniqueness for $H$ in $\Omega$ is also a set of uniqueness for $H H$. Then the $m$-AAK property for $H$ implies the compression property for $s_{m}$. In the case $m=0$ the converse is also true $[\mathrm{Ag} 1]$, but when $m>0$ it is not so.

Example 1.6. Let $0<\alpha<1$, let $\Omega=\{-\alpha, 0, \alpha\}$ and let $H$ be the restriction of the Hardy space $H^{2}$ to $\Omega$, so that the reproducing kernel of the 3 -dimensional space $H$ is

$$
k(\lambda, \mu)=1 /(1-\lambda \mu), \quad \lambda, \mu \in \Omega .
$$

Define $\varphi:\{-\alpha, \alpha\} \rightarrow \mathbb{C}$ by $\varphi(\lambda)=1 / \lambda$. The Pick matrix corresponding to these data is

$$
\frac{1}{\alpha^{2}}\left[\begin{array}{cc}
-1 & 1 \\
1 & -1
\end{array}\right]
$$

which has one negative and one zero eigenvalue. However $\varphi$ cannot be extended to a 1-pseudomultiplier of $H$ with preservation of $s_{1}$. For suppose we take $\varphi(0)=\zeta$. A simple calculation shows that the $3 \times 3$ Pick matrix corresponding to the augmented data has determinant $4 / \alpha^{2}$, independently of $\zeta$. Since the Pick matrix is nonsingular we have $s_{1}(\varphi) \neq 1$. It cannot be that $s_{1}(\varphi)<1$ since a restriction of $\varphi$ has $s_{1}$ equal to 1 , and hence we have $s_{1}(\varphi)>1$. Thus $H$ does not have the 1-AAK property. However, $H$ has the compression property for $s_{1}$ (for example, Theorem 1.9 below).

We shall need the following special case of Theorem 3.1 of [GRSW].

Lemma 1.7. Let $G$ be an $n$-dimensional Hilbert space, let $E$ be a subspace of codimension 1 in $G$, let $A$ be a linear operator on $E$ and let $m$ be a non-negative integer. Let $g \in G \backslash E$ and define $A(c)$ on $G$ by

$$
A(c)(x+\lambda g)=A x+c \lambda g
$$


for $x \in E, \lambda \in \mathbb{C}$. Then

$$
\inf _{c \in \mathbb{C}} s_{m}(A(c))=\max \left\{s_{m}(A), s_{m}(\tilde{A})\right\}
$$

where $\tilde{A}$ is the compression of $A(c)$ to $g^{\perp}$.

Note that $\tilde{A}$ does not depend on $c$. It is well known that in the case $m=0$ the infimum is attained at some $c \in \mathbb{C}$ : this is a version of "Parrott's Lemma" (see for example [Ag1]). When $m>1$ the infimum need not be attained. The issue is discussed in much greater generality in [CG].

One of the two main results of this paper is a converse to Corollary 1.3, in other words, a statement that the compression property for $s_{m}$ implies the $m$-AAK property. We have just seen in Example 1.6 that this implication does not hold in complete generality. It transpires that there is a big difference here between finite and infinite $\Omega$. In the example, to preserve the property $s_{1}(\varphi)=1$, we really need to take $\varphi(0)$ to be $\infty$, or in other words, to define $\varphi(\lambda)$ to be $1 / \lambda$. In the case of finite $\Omega$ this results in the domain of $\varphi$ being the complement of a single point, hence not being a set of uniqueness. For most function spaces of interest on infinite domains the complement of a finite set will be a set of uniqeness, and so a pseudomultiplier can perfectly well have some "infinities".

Theorem 1.8. Let $(H, \Omega)$ be a Hilbert function space with non-singular kernel, let $m$ be a non-negative integer and suppose that every cofinite set in $\Omega$ is a set of uniqueness for $H$ and for $H H$. Then $H$ has the $m$-AAK property if and only if $H$ has the compression property for $s_{m}$.

Proof. The forward implication is a consequence of Corollary 1.3. Suppose that $H$ has the compression property for $s_{m}$. Consider distinct points $\lambda_{1}, \ldots, \lambda_{n} \in \Omega$ and any $z_{1}, \ldots, z_{n} \in \mathbb{C}$ such that the Pick matrix (0.1) has $m$ or fewer negative eigenvalues. We must construct an interpolating $m$-pseudomultiplier $\varphi$ such that $s_{m}(\varphi) \leq 1$. As we have seen, the operator $T$ on

$$
\mathcal{M} \stackrel{\text { def }}{=} \operatorname{span}\left\{k_{\lambda_{1}}, \ldots, k_{\lambda_{n}}\right\}, \quad T k_{\lambda_{j}}=\bar{z}_{j} k_{\lambda_{j}},
$$

satisfies $s_{m}(T) \leq 1$.

Let $\mathcal{F}$ denote the collection of finite sets $F$ satisfying $\left\{\lambda_{1}, \ldots, \lambda_{n}\right\} \subset F \subset \Omega$. Pick $F \in \mathcal{F}$ and $\varepsilon>0$. We shall construct

$$
\varphi_{F \varepsilon}: F \rightarrow \mathbb{C}
$$

such that $\varphi_{F \varepsilon}\left(\lambda_{j}\right)=z_{j}, 1 \leq j \leq n$, and $s_{m}\left(\varphi_{F \varepsilon}\right)<1+\varepsilon$. Write

$$
F=\left\{\lambda_{1}, \ldots, \lambda_{n}, \lambda_{n+1}, \ldots, \lambda_{N}\right\}
$$

and let $\mathcal{M}_{F}=\operatorname{span}\left\{k_{\lambda}: \lambda \in F\right\}$. Further, let $\tilde{T}, \tilde{\mathcal{M}}$ be as in Definition 1.5 with $\alpha=\lambda_{n+1}$. By the hypothesis that $H$ have the compression property for $s_{m}$,

$$
s_{m}(\tilde{T}) \leq s_{m}(T) \leq 1 .
$$

By Lemma 1.7, if we define $T(c)$ on

$$
\mathcal{M}_{n+1} \stackrel{\text { def }}{=} \mathcal{M}_{\left\{\lambda_{1}, \ldots, \lambda_{n+1}\right\}}
$$

by

$$
T(c) k_{\lambda_{j}}= \begin{cases}\bar{z}_{j} k_{\lambda_{j}}, & 1 \leq j \leq n, \\ \bar{c} k_{\lambda_{n+1}}, & j=n+1,\end{cases}
$$


then

$$
\inf _{c} s_{m}(T(c))=\max \left\{s_{m}(T), s_{m}(\tilde{T})\right\} \leq 1 .
$$

Choose $c \in \mathbb{C}$ such that $s_{m}(T(c)) \leq 1+\varepsilon / 2$ and let $z_{n+1}(\varepsilon)=c$. Write $T(c)=T_{n+1}$ on $\mathcal{M}_{n+1}$, so that $s_{m}\left(T_{n+1}\right) \leq 1+\varepsilon / 2$. By repeating the argument we can find $z_{n+2}(\varepsilon) \in \mathbb{C}$ such that $s_{m}\left(T_{n+2}\right) \leq 1+\frac{\varepsilon}{2}+\frac{\varepsilon}{2^{2}}$. Continuing inductively we get a sequence $z_{j}(\varepsilon), n+1 \leq j \leq N$, such that the corresponding operators $T_{j}, n+1 \leq$ $j \leq N$, all satisfy

$$
s_{m}\left(T_{j}\right) \leq 1+\varepsilon .
$$

Define $\varphi_{F \varepsilon}\left(\lambda_{j}\right)$ to be $z_{j}$ for $1 \leq j \leq n$ and $z_{j}(\varepsilon)$ for $n+1 \leq j \leq N$, and define $T_{F \varepsilon}$ to be $T_{N}$. Then for each $F \in \mathcal{F}$ and $\varepsilon>0$ we have $\varphi_{F \varepsilon}: F \rightarrow \mathbb{C}$ and an operator $T_{F \varepsilon}$ on $\mathcal{M}_{F}$ such that $s_{m}\left(\varphi_{F \varepsilon}\right)=s_{m}\left(T_{F \varepsilon}\right) \leq 1+\varepsilon$ and $\varphi_{F \varepsilon}\left(\lambda_{j}\right)=z_{j}, 1 \leq j \leq n$. The idea, roughly speaking, is to construct an interpolating $m$-pseudomultiplier $\varphi$ by taking the pointwise limit of $\varphi_{F \varepsilon}$ as $F \rightarrow \Omega$ and $\varepsilon \rightarrow 0$.

We wish to define an ultrafilter on $\mathcal{F} \times(0,1)$. For each $F \in \mathcal{F}$ and $\varepsilon>0$ let

$$
Q(F, \varepsilon)=\{(G, x): G \in \mathcal{F}, F \subset G, 0<x<\varepsilon\} .
$$

$Q(F, \varepsilon)$ is a subset of $\mathcal{F} \times(0,1)$, and

$$
Q(F, \varepsilon) \cap Q\left(F^{\prime}, \varepsilon^{\prime}\right)=Q\left(F \cup F^{\prime}, \min \left\{\varepsilon, \varepsilon^{\prime}\right\}\right) .
$$

Thus the sets $Q(F, \varepsilon)$ constitute a filter base on $\mathcal{F} \times(0,1)$ as $F$ ranges over $\mathcal{F}$ and $\varepsilon$ ranges over $(0,1)$. Pick an ultrafilter $\mathcal{U}$ on $\mathcal{F} \times(0,1)$ which refines this filter base.

Define $\hat{\varphi}: \Omega \rightarrow \mathbb{C} \cup\{\infty\}$ by

$$
\hat{\varphi}(\lambda)=\lim _{\mathcal{U}} \varphi_{F \varepsilon}(\lambda), \quad \text { all } \lambda \in \Omega .
$$

That is, for fixed $\lambda$, we define $\hat{\varphi}(\lambda)$ to be the unique limit point in the compact Hausdorff space $\mathbb{C} \cup\{\infty\}$ of the ultrafilter on $\mathbb{C} \cup\{\infty\}$ consisting of those subsets $A$ for which

$$
\left\{(F, \varepsilon): F \in \mathcal{F}, \varepsilon>0, \varphi_{F \varepsilon}(\lambda) \in A\right\}
$$

belongs to $\mathcal{U}$. Now let

$$
\mathcal{P}=\{\lambda \in \Omega: \hat{\varphi}(\lambda)=\infty\},
$$

the set of singularities of $\hat{\varphi}$. We shall see shortly that $\mathcal{P}$ contains at most $m$ points. Let $\varphi$ be the restriction of $\hat{\varphi}$ to $\Omega \backslash \mathcal{P}$. We wish to show that $\varphi$ is the desired interpolating pseudomultiplier with $s_{m}(\varphi) \leq 1$.

Certainly $\varphi\left(\lambda_{j}\right)=z_{j}$ for $1 \leq j \leq n$, since $\varphi_{F \varepsilon}\left(\lambda_{j}\right)=z_{j}$ for all $F \in \mathcal{F}$ and $\varepsilon \in(0,1)$. By the spectral theorem, since $s_{m}\left(T_{F \varepsilon}\right) \leq 1+\varepsilon$, there exists a positive operator $B_{F \varepsilon} \leq(1+\varepsilon)^{2} 1$ and vectors $u_{F \varepsilon}^{0}, \ldots, u_{F \varepsilon}^{m-1} \in \mathcal{M}_{F}$ such that

$$
T_{F \varepsilon}^{*} T_{F \varepsilon}=B_{F \varepsilon}+\sum_{j=0}^{m-1} u_{F \varepsilon}^{j} \otimes u_{F \varepsilon}^{j}
$$

where $B_{F \varepsilon} u_{F \varepsilon}^{j}=0, \quad 0 \leq j \leq m-1$. For $\lambda \in F$ we have

$$
T_{F \varepsilon} k_{\lambda}=\bar{\varphi}_{F \varepsilon}(\lambda) k_{\lambda}
$$

and hence, for $\lambda, \mu \in F$,

$$
\bar{\varphi}_{F \varepsilon}(\lambda) \varphi_{F \varepsilon}(\mu)\left\langle k_{\lambda}, k_{\mu}\right\rangle=\left\langle B_{F \varepsilon} k_{\lambda}, k_{\mu}\right\rangle+\sum_{j=0}^{m-1} \bar{u}_{F \varepsilon}^{j}(\lambda) u_{F \varepsilon}^{j}(\mu) .
$$


In particular, for $\lambda \in F$ we have

$$
\begin{aligned}
\sum_{j=0}^{m-1}\left|u_{F \varepsilon}^{j}(\lambda)\right|^{2} & =\left|\varphi_{F \varepsilon}(\lambda)\right|^{2}\left\|k_{\lambda}\right\|^{2}-\left\langle B_{F \varepsilon} k_{\lambda}, k_{\lambda}\right\rangle \\
& \leq\left|\varphi_{F \varepsilon}(\lambda)\right|^{2}\left\|k_{\lambda}\right\|^{2} .
\end{aligned}
$$

Thus, if we define $g_{F \varepsilon}^{j}(\lambda)$ for $\lambda \in F, 0 \leq j \leq m-1$, by

$$
g_{F \varepsilon}^{j}(\lambda)= \begin{cases}u_{F \varepsilon}^{j}(\lambda) / \varphi_{F \varepsilon}(\lambda) & \text { if } \varphi_{F \varepsilon}(\lambda) \neq 0 \\ 0 & \text { if } \varphi_{F \varepsilon}(\lambda)=0\end{cases}
$$

then we obtain

$$
\left|g_{F \varepsilon}^{j}(\lambda)\right| \leq\left\|k_{\lambda}\right\|, \quad 0 \leq j \leq m-1
$$

and

$$
u_{F \varepsilon}^{j}=\varphi_{F \varepsilon} g_{F \varepsilon}^{j} \text { on } F .
$$

On dividing through by $\bar{\varphi}_{F \varepsilon}(\lambda) \varphi_{F \varepsilon}(\mu)$ in (1.8) we have, for $\lambda, \mu \in \Omega$ such that $\bar{\varphi}_{F \varepsilon}(\lambda) \varphi_{F \varepsilon}(\mu) \neq 0$,

$$
\left\langle k_{\lambda}, k_{\mu}\right\rangle=\frac{1}{\bar{\varphi}_{F \varepsilon}(\lambda) \varphi_{F \varepsilon}(\mu)}\left\langle B_{F \varepsilon} k_{\lambda}, k_{\mu}\right\rangle+\sum_{j=0}^{m-1} \bar{g}_{F \varepsilon}^{j}(\lambda) g_{F \varepsilon}^{j}(\mu) .
$$

For $\lambda \in \Omega$ and $0 \leq j \leq m-1$ let

$$
a_{j}(\lambda)=\lim _{\mathcal{U}} g_{F \varepsilon}^{j}(\lambda) .
$$

Then $\left|a_{j}(\lambda)\right| \leq\left\|k_{\lambda}\right\|$ for all $\lambda \in \Omega$. By weak compactness $\lim _{\mathcal{U}} B_{F \varepsilon}$ exists in the weak operator topology. Call this limit $B$ and note that $\|B\| \leq 1+\varepsilon$ for all $\varepsilon>0$, so that $\|B\| \leq 1$. Take limits along $\mathcal{U}$ in (1.9) to obtain, for all $\lambda, \mu \in \Omega$ such that $\varphi(\lambda) \neq 0$ and $\varphi(\mu) \neq 0$,

$$
\left\langle k_{\lambda}, k_{\mu}\right\rangle=\frac{1}{\bar{\varphi}(\lambda) \varphi(\mu)}\left\langle B k_{\lambda}, k_{\mu}\right\rangle+\sum_{j=0}^{m-1} \bar{a}_{j}(\lambda) a_{j}(\mu),
$$

and note that this relation remains valid (with the natural interpretation) if $\hat{\varphi}(\lambda)$ or $\hat{\varphi}(\mu)=\infty$. In particular, for $\lambda, \mu \in \mathcal{P}$,

$$
\left\langle k_{\lambda}, k_{\mu}\right\rangle=\sum_{j=0}^{m-1} \bar{a}_{j}(\lambda) a_{j}(\mu) .
$$

This implies that the matrix $[k(\mu, \lambda)]_{\lambda, \mu \in \mathcal{P}}$ has rank at most $m$. In view of the non-singularity of $k$, there can be at most $m$ points in $\mathcal{P}$.

Let $\mathcal{R}$ be the closed linear span of $\left\{k_{\lambda}: \lambda \in \Omega, \varphi(\lambda) \neq 0\right\}$. The relation (1.10) implies that we can define an isometry $V: \mathcal{R} \rightarrow H \oplus \mathbb{C}^{m}$ by

$$
V k_{\lambda}= \begin{cases}\bar{\varphi}(\lambda)^{-1} B^{\frac{1}{2}} k_{\lambda} \oplus\left(\bar{a}_{0}(\lambda), \ldots, \bar{a}_{m-1}(\lambda)\right) & \text { if } \hat{\varphi}(\lambda) \neq 0 \text { or } \infty \\ 0 \oplus\left(\bar{a}_{0}(\lambda), \ldots, \bar{a}_{m-1}(\lambda)\right) & \text { if } \hat{\varphi}(\lambda)=\infty\end{cases}
$$

Write $V$ in the form

$$
V=\left[\begin{array}{c}
C \\
1 \otimes f_{0} \\
\vdots \\
1 \otimes f_{m-1}
\end{array}\right]
$$


for some $f_{0}, \ldots, f_{m-1} \in \mathcal{R}$. Since $V^{*} V=1$,

$$
C^{*} C+f_{0} \otimes f_{0}+\cdots+f_{m-1} \otimes f_{m-1}=1 .
$$

By (1.11), for all $\lambda$ such that $\varphi(\lambda) \neq 0$,

$$
\begin{aligned}
C k_{\lambda} & = \begin{cases}\bar{\varphi}(\lambda)^{-1} B^{\frac{1}{2}} k_{\lambda} & \text { if } \hat{\varphi}(\lambda) \neq \infty, \\
0 & \text { if } \hat{\varphi}(\lambda)=\infty,\end{cases} \\
f_{j}(\lambda) & =a_{j}(\lambda) .
\end{aligned}
$$

Define $C^{\sharp}: H=\mathcal{R} \oplus \mathcal{R}^{\perp} \rightarrow H$ to be $C$ on $\mathcal{R}$ and 0 on $\mathcal{R}^{\perp}$. Let $L=B^{\frac{1}{2}} C^{\sharp} \in \mathcal{L}(H)$. By (1.12), if $\hat{\varphi}(\lambda) \neq 0$ or $\infty$,

$$
\begin{aligned}
L^{*} k_{\lambda} & =\left(C^{\sharp}\right)^{*} B^{\frac{1}{2}} k_{\lambda}=\left(C^{\sharp}\right)^{*} \bar{\varphi}(\lambda) C k_{\lambda} \\
& =\bar{\varphi}(\lambda) C^{*} C k_{\lambda}=\bar{\varphi}(\lambda)\left(1-\sum_{j=0}^{m-1} f_{j} \otimes f_{j}\right) k_{\lambda} .
\end{aligned}
$$

This relation remains valid when $\varphi(\lambda)=0$. Indeed, it follows from taking limits along $\mathcal{U}$ in (1.8) that $\varphi(\lambda)=0$ implies $B^{\frac{1}{2}} k_{\lambda}=0$ and hence that $L^{*} k_{\lambda}=$ $\left(C^{\sharp}\right)^{*} B^{\frac{1}{2}} k_{\lambda}=0$. Thus, for all $\lambda \in \Omega \backslash \mathcal{P}$,

$$
L^{*} k_{\lambda}=\bar{\varphi}(\lambda)(1-A) k_{\lambda}
$$

where $A$ is the Hermitian contraction

$$
A=f_{0} \otimes f_{0}+\cdots+f_{m-1} \otimes f_{m-1}
$$

of rank at most $m$. Dualizing this relation we obtain, for all $h \in H$ and $\lambda \in \Omega \backslash \mathcal{P}$,

$$
(L h)(\lambda)=\varphi(\lambda)(h(\lambda)-A h(\lambda)) .
$$

Ker $A$ is a closed subspace of $H$ of codimension at most $m$, and for $h \in \operatorname{Ker} A$ we have

$$
\varphi h|\Omega \backslash \mathcal{P}=L h| \Omega \backslash \mathcal{P} \in H \mid \Omega \backslash \mathcal{P}
$$

and

$$
\|L h\|=\left\|B^{\frac{1}{2}} C^{*} h\right\| \leq\|h\| .
$$

Thus $\varphi$ multiplies a closed $m$-codimensional subspace of $H$ contractively into $H \mid \Omega \backslash \mathcal{P}$, and so $s_{m}(\varphi) \leq 1$.

The function $\varphi$ is almost the $m$-pseudomultiplier we are trying to construct. Its domain is the set $D \stackrel{\text { def }}{=} \Omega \backslash \mathcal{P}$, which contains all but at most $m$ points of $\Omega$ and so by assumption is a set of uniqueness for $H$. It is defined at each $\lambda_{i}$ and satisfies $\varphi\left(\lambda_{i}\right)=z_{i}, 1 \leq i \leq n$. All that remains is to ensure that the points in the complement of the domain of $\varphi$ are pseudopoles of $\varphi$.

Consider $\mu \in \mathcal{P}$. We claim that either $\mu$ is a pseudopole of $\varphi$ or we can define $\varphi(\mu)$ to be a (finite) number so as to preserve the relation

$$
\operatorname{Lh}(\lambda)=\varphi(\lambda) h(\lambda)
$$

for all $\lambda$ in the domain of $\varphi$ and all $h \in \operatorname{Ker} A$. There are three cases.

Case 1. Suppose $k_{\mu} \notin A H$. Then there exists $h \in \operatorname{Ker} A$ such that $h(\mu) \neq 0$. Define $\varphi(\mu)$ to be $L h(\mu) / h(\mu)$. This formula does define $\varphi(\mu)$ uniquely, for if $h_{1}, h_{2}$ are two elements of $\operatorname{Ker} A$ which do not vanish at $\mu$, then we have, from (1.14),

$$
h_{1}(\lambda) \cdot L h_{2}(\lambda)=h_{1}(\lambda) \varphi(\lambda) h_{2}(\lambda)=L h_{1}(\lambda) \cdot h_{2}(\lambda)
$$


for all $\lambda \in \Omega \backslash \mathcal{P}$. Since $\Omega \backslash \mathcal{P}$ is a set of uniqueness for $H H$ it follows that $L h_{1} \cdot h_{2}=h_{1} \cdot L h_{2}$, and so $L h_{1} / h_{1}$ and $L h_{2} / h_{2}$ agree at $\mu$. Clearly (1.14) remains true for $\lambda=\mu$.

Case 2. Suppose $k_{\mu} \in A H$ and $L \operatorname{Ker} A \subset k_{\mu}^{\perp}$. Then for any $h \in \operatorname{Ker} A$ we have $h(\mu)=0$ and $L h(\mu)=0$, so that (1.14) remains true for any choice of $\varphi(\mu)$.

Case 3. Suppose $k_{\mu} \in A H$ and $L \operatorname{Ker} A \not \subset k_{\mu}^{\perp}$. Then there exists $h \in \operatorname{Ker} A \subset k_{\mu}^{\perp}$ such that $h(\mu)=0$ and $\varphi h$ extends to $L h \in H$ which does not vanish at $\mu$. That is, $\mu$ is a pseudopole of $\varphi$. This establishes the claim.

For each $\mu \in \mathcal{P}$ which is not a pseudopole of $\varphi$ assign $\varphi(\mu)$ an appropriate finite value to preserve (1.14). Then $\varphi$ is the desired interpolating $m$-pseudomultiplier such that $s_{m}(\varphi) \leq 1$. It follows that $H$ has the $m$-AAK property.

The last result contains the theorem of Akhiezer, Adamyan, Arov and Krein, which is obtained on taking $H=H^{2}, \Omega=\mathbb{D}$ in the following statement.

Theorem 1.9. Let $k$ be a Szegö kernel on a set $\Omega$ and let $H$ be the corresponding Hilbert space of functions. Then $(H, \Omega)$ has the compression property for $s_{m}$ for every non-negative integer $m$. Moreover, if every cofinite subset of $\Omega$ is a set of uniqueness for $H$ and $H H$, then $(H, \Omega)$ has the $m$-AAK property for every nonnegative integer $m$.

Proof. Consider distinct points $\lambda_{1}, \ldots, \lambda_{n} \in \Omega$ and $z_{1}, \ldots, z_{n} \in \mathbb{C}$. Let $\mathcal{M}, \tilde{\mathcal{M}}, T$ and $\tilde{T}$ be as in Definition 1.5. We must show that $s_{m}(\tilde{T}) \leq s_{m}(T)$. By hypothesis there exist functions $a: \Omega \rightarrow \mathbb{C}, b: \Omega \rightarrow \mathbb{D}$ such that $b$ is injective and

$$
k(\lambda, \mu)=\frac{a(\lambda) \bar{a}(\mu)}{1-b(\lambda) \bar{b}(\mu)} .
$$

We have, for $\lambda=\lambda_{i}, 1 \leq i \leq n$,

$$
\tilde{k}_{\lambda}=P_{\tilde{\mathcal{M}}} k_{\lambda}=k_{\lambda}-\frac{\left\langle k_{\lambda}, k_{\alpha}\right\rangle}{k(\alpha, \alpha)} k_{\alpha}
$$

A straightforward calculation now gives

$$
\tilde{k}_{\lambda}(\mu)=c_{\alpha}(\mu) k_{\lambda}(\mu) \bar{c}_{\alpha}(\lambda)
$$

where

$$
c_{\alpha}(\mu)=\frac{b(\alpha)-b(\mu)}{1-\bar{b}(\alpha) b(\mu)} .
$$

Note that $c_{\alpha}(\cdot)$ is non-zero on $\left\{\lambda_{1}, \ldots, \lambda_{n}\right\}$ since $\alpha$ is taken to be distinct from each $\lambda_{i}$. Define $U: \mathcal{M} \rightarrow \tilde{\mathcal{M}}$ by

$$
U k_{i}=\bar{c}_{\alpha}\left(\lambda_{i}\right)^{-1} \tilde{k}_{i}
$$

where $k_{i}$ denotes $k_{\lambda_{i}}$. The relation (1.15) says precisely that

$$
\left\langle U k_{i}, U k_{j}\right\rangle=\left\langle k_{i}, k_{j}\right\rangle
$$

that is, $U$ is unitary. Moreover

$$
\begin{aligned}
\tilde{T} U k_{j} & =\bar{c}_{\alpha}\left(\lambda_{j}\right)^{-1} \tilde{T} \tilde{k}_{j}=c_{\alpha}\left(\lambda_{j}\right)^{-1} \bar{z}_{j} \tilde{k}_{j} \\
& =\bar{z}_{j} U k_{j},
\end{aligned}
$$

so that $\tilde{T} U=U T$. Hence $\tilde{T}$ is unitarily equivalent to $T$, and so $s_{m}(\tilde{T})=s_{m}(T)$. That is, $H$ has the compression property for $s_{m}$. The second assertion is immediate from Theorem 1.8. 


\section{SZEGÖ KERNELS}

One of the main questions we address is: what converses of the Adamyan-ArovKrein theorem hold? That is, if $H$ is a Hilbert function space, does the validity of AAK-type extension properties force the reproducing kernel of $H$ to be a Szegö kernel? In this section we establish some characterizations of Szegö kernels. A kernel $k$ on a set $\Omega$ is a complex-valued function on $\Omega \times \Omega$ which is Hermitian symmetric, i.e. satisifes $k(\mu, \lambda)^{-}=k(\lambda, \mu)$ for all $\lambda, \mu \in \Omega$.

Recall that in this paper we are adopting a narrow definition of Szegö kernel: a kernel $k$ on a set $\Omega$ is Szegö if there exist functions

$$
a: \Omega \rightarrow \mathbb{C} \backslash\{0\}, \quad b: \Omega \rightarrow \mathbb{D}
$$

such that $b$ is injective and

$$
k(\lambda, \mu)=\frac{a(\lambda) \bar{a}(\mu)}{1-b(\lambda) \bar{b}(\mu)}, \quad \lambda, \mu \in \Omega .
$$

Note that a Szegö kernel does not vanish at any point of $\Omega \times \Omega$. Such a kernel $k$ is indeed positive and non-singular; this is a simple consequence of the fact that the standard Szegö kernel $(1-\lambda \bar{\mu})^{-1}$ on $\mathbb{D}$ has these properties. Let us define the rank of a Hermitian symmetric function $F: \Omega \times \Omega \rightarrow \mathbb{C}$ to be the dimension of the "column space", that is, the dimension of the subspace $\operatorname{span}\{F(\cdot, \mu): \mu \in \Omega\}$ of $\mathbb{C}^{\Omega}$. If $k$ is the reproducing kernel of a Hilbert function space $H$ on a set $\Omega$ and $\alpha \in \Omega$, we denote by $k^{\alpha}$ the reproducing kernel of the subspace $k_{\alpha}^{\perp}$ of $H$ :

$$
k^{\alpha}(\lambda, \mu)=k(\lambda, \mu)-\frac{k(\lambda, \alpha) k(\alpha, \mu)}{k(\alpha, \alpha)} .
$$

Lemma 2.1. Let $k$ be a non-singular positive kernel on a set $\Omega$ containing at least 2 points and suppose $k$ does not vanish at any point of $\Omega \times \Omega$. The following are equivalent:

(1) $k$ is a Szegő kernel;

(2) for some $\alpha \in \Omega, k^{\alpha} / k$ has rank one;

(3) for every $\alpha \in \Omega, k^{\alpha} / k$ has rank one;

(4) $1 / k$ has rank two.

Proof. (1) $\Rightarrow(3)$ It is a straightforward calculation that if $k$ is given by (2), then

$$
k^{\alpha}(\lambda, \mu)=\varphi(\lambda) k(\lambda, \mu) \bar{\varphi}(\mu)
$$

where

$$
\varphi(\lambda)=\frac{b(\lambda)-b(\alpha)}{1-\bar{b}(\alpha) b(\lambda)}
$$

whence $k^{\alpha} / k$ has rank one.

$(3) \Rightarrow(2)$ is immediate.

$(2) \Rightarrow(4)$ It follows from (2) that there exists a function $\varphi: \Omega \rightarrow \mathbb{C}$ such that

$$
1-\frac{k(\lambda, \alpha) k(\alpha, \mu)}{k(\lambda, \mu) k(\alpha, \alpha)}=\varphi(\lambda) \bar{\varphi}(\mu)
$$

for all $\lambda, \mu \in \Omega$. Hence

$$
\frac{1}{k(\lambda, \mu)}=\frac{k(\alpha, \alpha)}{k(\lambda, \alpha) k(\alpha, \mu)}(1-\varphi(\lambda) \bar{\varphi}(\mu))
$$


and so the column space of $1 / k$ is spanned by the functions $1 / k(\cdot, \alpha)$ and $\varphi / k(\cdot, \alpha)$. The rank of $1 / k$ is thus at most 2 . If the rank were 1 , then $k$ would also have rank 1 , and since $\Omega$ contains more than one point the non-singularity of $k$ would be contradicted. Thus the rank of $1 / k$ is 2 .

$(4) \Rightarrow(1)$ Suppose that the column space of $1 / k$ is spanned by linearly independent functions $\varphi, \psi$. Note that since $k$ is positive and non-singular, any $2 \times 2$ principal submatrix of $1 / k$ has negative determinant, and hence $1 / k$ cannot be a positive kernel; nor can it be a negative kernel, since it takes positive values on the diagonal of $\Omega \times \Omega$. By hypothesis, there exist functions $u, v$ on $\Omega$ such that

$$
\frac{1}{k(\lambda, \mu)}=\varphi(\lambda) \bar{u}(\mu)+\psi(\lambda) \bar{v}(\mu), \quad \lambda, \mu \in \Omega
$$

By Hermitian symmetry we have also

$$
\frac{1}{k(\lambda, \mu)}=u(\lambda) \bar{\varphi}(\mu)+v(\lambda) \bar{\psi}(\mu)
$$

Since $\bar{\varphi}$ and $\bar{\psi}$ are linearly independent, $u$ and $v$ lie in the column space of $1 / k$, say

$$
u=\overline{c_{1}} \varphi+\bar{c}_{2} \psi, \quad v=\bar{d}_{1} \varphi+\bar{d}_{2} \psi .
$$

We therefore have

$$
\frac{1}{k(\lambda, \mu)}=\left[\begin{array}{ll}
\bar{\varphi}(\mu) & \bar{\psi}(\mu)
\end{array}\right]\left[\begin{array}{ll}
c_{1} & d_{1} \\
c_{2} & d_{2}
\end{array}\right]\left[\begin{array}{l}
\varphi(\lambda) \\
\psi(\lambda)
\end{array}\right] .
$$

The $2 \times 2$ matrix on the right hand side is Hermitian and therefore can be factorized as $P^{*} \operatorname{diag}\{ \pm 1, \pm 1\} P$ for some non-singular matrix $P=\left[p_{i j}\right]$. Replacing $\varphi, \psi$ by $p_{11} \varphi+p_{12} \psi$ and $p_{21} \varphi+p_{22} \psi$ respectively, we have

$$
\frac{1}{k(\lambda, \mu)}= \pm \varphi(\lambda) \bar{\varphi}(\mu) \pm \psi(\lambda) \bar{\psi}(\mu) \text {. }
$$

Since $1 / k$ can be neither positive nor negative as a kernel, there must be one plus and one minus and we have

$$
\frac{1}{k(\lambda, \mu)}=\varphi(\lambda) \bar{\varphi}(\mu)-\psi(\lambda) \bar{\psi}(\mu) .
$$

In particular, for any $\lambda \in \Omega$,

$$
|\varphi(\lambda)|^{2}-|\psi(\lambda)|^{2}=\frac{1}{k(\lambda, \lambda)}>0
$$

so that $\varphi$ does not vanish on $\Omega$ and $|\psi / \varphi|<1$. Let $a=1 / \varphi, b=\psi / \varphi$. Then $a, b$ map $\Omega$ into $\mathbb{C} \backslash\{0\}, \mathbb{D}$ respectively, and

$$
k(\lambda, \mu)=\frac{1}{\varphi(\lambda) \bar{\varphi}(\mu)-\psi(\lambda) \bar{\psi}(\mu)}=\frac{a(\lambda) \bar{a}(\mu)}{1-b(\lambda) \bar{b}(\mu)}
$$

for any $\lambda, \mu \in \Omega$. Since $k$ is non-singular, $b$ is necessarily injective, and so $k$ is a Szegö kernel.

It is clear that any restriction of a Szegö kernel is again Szegö. In order to determine whether a given kernel is Szegö it is enough to check restrictions to small subsets.

Lemma 2.2. Let $k$ be a non-singular positive kernel on a set $\Omega$ containing at least 4 points. If every restriction of $k$ to a 4-point subset of $\Omega$ is Szegö, then $k$ is Szegö. 
Proof. Fix $\alpha \in \Omega$. The hypothesis implies that $k$ does not vanish on $\Omega \times \Omega$, and hence we can define a kernel $F$ on $\Omega \backslash\{\alpha\}$ by $F=k^{\alpha} / k$. By Lemma 2.1, the restriction of $F$ to any 3-point subset of $\Omega \backslash\{\alpha\}$ has rank 1. Suppose $k$ is not Szegö: then there exist $\mu_{1}, \mu_{2} \in \Omega \backslash\{\alpha\}$ such that $F\left(\cdot, \mu_{1}\right),\left(F \cdot, \mu_{2}\right)$ are linearly independent. Observe that if $f_{1}, f_{2}$ are linearly independent functions and $x_{1}$ is a point such that $f_{1}\left(x_{1}\right) \neq 0$, then there is some point $x_{2}$ such that $\left[f_{i}\left(x_{j}\right)\right]_{i, j=1}^{2}$ has rank 2. Apply this observation to $F\left(\cdot, \mu_{1}\right), F\left(\cdot, \mu_{2}\right)$ with $x_{1}=\mu_{1}$ : we can do this since $F\left(\mu_{1}, \mu_{1}\right) \neq 0$, else we should have $k\left(\mu_{1}, \mu_{1}\right) k(\alpha, \alpha)=\left|k\left(\mu_{1}, \alpha\right)\right|^{2}$, contradicting the non-singularity of $k$. Hence there exists $\mu_{3} \in \Omega \backslash\{\alpha\}$ such that

$$
\left[\begin{array}{ll}
F\left(\mu_{1}, \mu_{1}\right) & F\left(\mu_{1}, \mu_{2}\right) \\
F\left(\mu_{3}, \mu_{1}\right) & F\left(\mu_{3}, \mu_{2}\right)
\end{array}\right]
$$

has rank 2. This is a submatrix of $\left[F\left(\mu_{i}, \mu_{j}\right)\right]_{i, j=1}^{3}$, which accordingly has rank at least 2 , a contradiction.

It is plausible to guess that, in order to show a kernel is Szegö, it suffices to show that restrictions to 3 -point subsets are Szegö. The following example shows it does not.

Example 2.3. Let $\alpha>2+\sqrt{5}$ and let

$$
K=\left[\begin{array}{cccc}
\alpha^{2} & \alpha & \alpha & 1 \\
\alpha & \frac{\alpha+1}{2} & \frac{\alpha+1}{2 \alpha} & 1 \\
\alpha & \frac{\alpha+1}{2 \alpha} & \frac{\alpha+1}{2} & 1 \\
1 & 1 & 1 & 1
\end{array}\right]
$$

It may be verified that $K>0$ and that every principal $3 \times 3$ submatrix of $1 / K$ is singular, but

$$
\operatorname{det}(1 / K)=\frac{4(\alpha-1)^{3}}{\alpha^{2}(\alpha+1)} \neq 0
$$

That is, every 3-point restriction of $K$ is Szegö, but $K$ is not itself Szegö.

\section{Characterisation of COMpression Properties}

Theorem 1.8 reduces the study of the AAK property to concrete questions about kernels on finite sets, and so enables us to find the kernels with the desired properties by elementary algebra. Let us investigate the compression property for $s_{1}$ in the case of a Hilbert space $H$ of functions on a set $\Omega$ containing a small number of points. If $\Omega$ has only one or two points the property is trivial.

Theorem 3.1. Let $H$ be a Hilbert space of functions on a three-point set $\Omega$ with non-singular kernel $k$. Then $H$ has the compression property for $s_{1}$ if and only if

$$
\left(\prod_{i, j=1}^{3} k_{i j}\right) \operatorname{det}\left[\frac{1}{k_{i j}}\right]_{i, j=1}^{3} \leq 0,
$$

while $H$ has the compression property for $s_{0}$ if and only if

$$
\left(\prod_{i, j=1}^{3} k_{i j}\right) \operatorname{det}\left[\frac{1}{k_{i j}}\right]_{i, j=1}^{3} \geq 0 .
$$


Note. The left hand sides in these two conditions are to be understood as polynomials in the $k_{i j}$, so that the statements are meaningful and valid even if some $k_{i j}$ are zero. Indeed, one can write this polynomial as

$$
\left|\begin{array}{lll}
k_{12} k_{13} & k_{11} k_{13} & k_{11} k_{12} \\
k_{22} k_{23} & k_{21} k_{23} & k_{21} k_{22} \\
k_{32} k_{33} & k_{31} k_{33} & k_{31} k_{32}
\end{array}\right| .
$$

If all $k_{i j}$ are non-zero, then $\prod k_{i j}>0$ and so (3.1) is equivalent to $\operatorname{det}\left[1 / k_{i j}\right] \leq 0$.

We shall establish a number of equivalent conditions for the compression property. As is implicit in the above statement, we are assuming that $\Omega=\{1,2,3\}$ and are writing $k_{i j}$ for $k(i, j)$. Let $K$ denote the matrix $\left[k_{i j}\right]_{i, j=1}^{3}$ and $1 / K$ the matrix $\left[1 / k_{i j}\right]_{i, j=1}^{3}$.

Lemma 3.2. The following conditions are equivalent.

(1) $H$ has the compression property for $s_{1}$;

(2) if

$$
A=\left[k_{i j}\right]_{i, j=1}^{2}, \quad \tilde{A}=\left[k_{i j}-\frac{k_{i 3} k_{3 j}}{k_{33}}\right]_{i, j=1}^{2}
$$

then

$$
A * A^{-T} \leq \tilde{A} * \tilde{A}^{-T}
$$

(3) $k_{11} k_{22} k_{33} \operatorname{det} K \leq\left(k_{11} k_{33}-\left|k_{13}\right|^{2}\right)\left(k_{11} k_{22}-\left|k_{12}\right|^{2}\right)\left(k_{22} k_{33}-\left|k_{23}\right|^{2}\right)$;

(4) $\left(\prod_{i, j=1}^{3} k_{i j}\right) \operatorname{det}(1 / K) \leq 0$.

Moreover, the following conditions are also equivalent.

$\left(1^{\prime}\right) H$ has the compression property for $s_{0}$;

$\left(2^{\prime}\right)$ for $A, \tilde{A}$ as in (2) above,

$$
A * A^{-T} \geq \tilde{A} * \tilde{A}^{-T}
$$

$\left(3^{\prime}\right) k_{11} k_{22} k_{33} \operatorname{det} K \geq\left(k_{22} k_{33}-\left|k_{23}\right|^{2}\right)\left(k_{11} k_{33}-\left|k_{13}\right|^{2}\right)\left(k_{11} k_{22}-\left|k_{12}\right|^{2}\right)$;

$\left(4^{\prime}\right)\left(\prod_{i, j=1}^{3} k_{i j}\right) \operatorname{det}(1 / K) \geq 0$.

The following conditions are also equivalent.

$\left(1^{\prime \prime}\right) H$ has the compression property for both $s_{0}$ and $s_{1}$;

$\left(2^{\prime \prime}\right)$ for $A, \tilde{A}$ as in (2) above,

$$
A * A^{-T}=\tilde{A} * \tilde{A}^{-T}
$$

$\left(3^{\prime \prime}\right) k_{11} k_{22} k_{33} \operatorname{det} K=\left(k_{22} k_{33}-\left|k_{23}\right|^{2}\right)\left(k_{11} k_{33}-\left|k_{13}\right|^{2}\right)\left(k_{11} k_{22}-\left|k_{12}\right|^{2}\right)$;

$\left(4^{\prime \prime}\right)\left(\prod_{i, j=1}^{3} k_{i j}\right) \operatorname{det}(1 / K)=0$

$\left(5^{\prime \prime}\right)$ there exists a non-singular diagonal matrix $\Lambda$ such that $K^{-T}=\Lambda K \Lambda^{*}$.

For this and subsequent theorems we need to calculate the characteristic polynomials of $T^{*} T$ and $\tilde{T} * \tilde{T}$, where $T$ and $\tilde{T}$ are the operators occurring in the definition of the compression property. 
Lemma 3.3. Let $\lambda_{1}, \ldots, \lambda_{n}, \alpha, z_{1}, \ldots, z_{n}, \mathcal{M}, \tilde{\mathcal{M}}, T$ and $\tilde{T}$ be as in Definition 1.5. The characteristic polynomials of $T^{*} T, \tilde{T}^{*} \tilde{T}$ are $p, \tilde{p}$ respectively, where

$$
\begin{gathered}
p(t)=\Delta^{-1} \operatorname{det}\left[\left(t-z_{i} \bar{z}_{j}\right) k\left(\lambda_{i}, \lambda_{j}\right)\right]_{i, j=1}^{n}, \\
\tilde{p}(t)=\tilde{\Delta}^{-1} \operatorname{det}\left[\left(t-z_{i} \bar{z}_{j}\right) \tilde{k}\left(\lambda_{i}, \lambda_{j}\right)\right]_{i, j=1}^{n}, \\
\Delta=\operatorname{det}\left[k\left(\lambda_{i}, \lambda_{j}\right)\right]_{i, j=1}^{n}, \quad \tilde{\Delta}=\operatorname{det}\left[\tilde{k}\left(\lambda_{i}, \lambda_{j}\right)\right]_{i, j=1}^{n}
\end{gathered}
$$

and

$$
\tilde{k}\left(\lambda_{i}, \lambda_{j}\right)=k\left(\lambda_{i}, \lambda_{j}\right)-\frac{k\left(\lambda_{i}, \alpha\right) k\left(\alpha, \lambda_{j}\right)}{k(\alpha, \alpha)} .
$$

Proof. Routine linear algebra shows that the matrix of $A \in \mathcal{L}(\mathcal{M})$ with respect to the basis $k_{\lambda_{1}}, \ldots, k_{\lambda_{n}}$ of $\mathcal{M}$ is

$$
K^{-1}\left[\left\langle A k_{\lambda_{j}}, k_{\lambda_{i}}\right\rangle\right]_{i, j=1}^{n}
$$

where

$$
K=\left[k\left(\lambda_{i}, \lambda_{j}\right)\right]_{i, j=1}^{n} .
$$

Indeed, the $(i, j)$-entry of the matrix of $A$ is $\left\langle A k_{\lambda_{j}}, w_{i}\right\rangle$ where $w_{1}, \ldots, w_{n}$ is the dual basis to $k_{\lambda_{1}}, \ldots, k_{\lambda_{n}}$. If we write $w_{i}=\sum_{r} \gamma_{i r} k_{\lambda_{r}}$, then we have

$$
\delta_{i j}=\left\langle k_{\lambda_{j}}, w_{i}\right\rangle=\sum_{r} \bar{\gamma}_{i r}\left\langle k_{\lambda_{j}}, k_{\lambda_{r}}\right\rangle=\sum_{r} \bar{\gamma}_{i r} k\left(\lambda_{r}, \lambda_{j}\right),
$$

that is, $\left[\bar{\gamma}_{i j}\right]_{i, j=1}^{n}=K^{-1}$, while

$$
\left\langle A k_{\lambda_{j}}, w_{i}\right\rangle=\sum_{r} \bar{\gamma}_{i r}\left\langle A k_{\lambda_{j}}, k_{\lambda_{r}}\right\rangle
$$

which is the $(i, j)$-entry of the matrix (3.2).

On applying this observation to $T^{*} T$ we find that

$$
T^{*} T \sim K^{-1}\left[\left\langle T k_{\lambda_{j}}, T k_{\lambda_{i}}\right\rangle\right]=K^{-1}\left[z_{i} \bar{z}_{j} k\left(\lambda_{i}, \lambda_{j}\right)\right],
$$

and hence

$$
\begin{aligned}
p(t) & =\operatorname{det}\left(t-T^{*} T\right) \\
& =\Delta^{-1} \operatorname{det}\left[\left(t-z_{i} \bar{z}_{j}\right) k\left(\lambda_{i}, \lambda_{j}\right)\right]
\end{aligned}
$$

as required. Similar reasoning gives the formula for $\tilde{p}$ (recall equations (1.4), (1.5)).

Proof of Lemma 3.2. We prove (1) $\Leftrightarrow(2)$. We have to consider subsets $\lambda_{1}, \ldots, \lambda_{n}$, $\alpha$ of distinct points of $\Omega=\{1,2,3\}$. If $n=1$, then both $T$ and $\tilde{T}$ in Definition 1.5 have rank one, and so $s_{1}(T)=s_{1}(\tilde{T})=0$. Take $n=2, \lambda_{1}=1, \lambda_{2}=2, \alpha=3$, and corresponding data values $z_{1}, z_{2} \in \mathbb{C}$. By the foregoing lemma the characteristic polynomial of $T^{*} T$ is

$$
\begin{aligned}
p(t) & =\Delta^{-1} \operatorname{det}\left[\left(t-z_{i} \bar{z}_{j}\right) k(i, j)\right]_{i, j=1}^{2} \\
& =t^{2}-\beta t+\left|z_{1} z_{2}\right|^{2},
\end{aligned}
$$

where we find, after a simple calculation, that

$$
\beta=\left\langle A * A^{-T} z, z\right\rangle_{\mathbb{C}^{2}} \text { where } z=\left[\begin{array}{ll}
z_{1} & z_{2}
\end{array}\right]^{T} .
$$


Hence

$$
s_{1}(T)^{2}=\frac{1}{2}\left(\beta-\sqrt{\beta^{2}-4\left|z_{1} z_{2}\right|^{2}}\right)
$$

and likewise

$$
s_{1}(\tilde{T})^{2}=\frac{1}{2}\left(\tilde{\beta}-\sqrt{\tilde{\beta}^{2}-4\left|z_{1} z_{2}\right|^{2}}\right)
$$

where

$$
\tilde{\beta}=\left\langle\tilde{A} * \tilde{A}^{-T} z, z\right\rangle_{\mathbb{C}^{2}} .
$$

On differentiating with respect to $\beta$ we find that $s_{1}(T)$ is a strictly decreasing function of $\beta$ on $\left(2\left|z_{1} z_{2}\right|, \infty\right)$, and so $s_{1}(\tilde{T}) \leq s_{1}(T)$ if and only if

$$
\left\langle A * A^{-T} z, z\right\rangle \leq\left\langle\tilde{A} * \tilde{A}^{-T} z, z\right\rangle .
$$

This holds for all $z_{1}, z_{2} \in \mathbb{C}$ if and only if

$$
A * A^{-T} \leq \tilde{A} * \tilde{A}^{-T} .
$$

It follows that $(1) \Rightarrow(2)$; conversely, (2) implies that $s_{1}(\tilde{T}) \leq s_{1}(T)$ whenever we take $\lambda_{1}=1, \lambda_{2}=2, \alpha=3$. We shall see later that the inequality remains valid when $\alpha=1$ or 2 .

A similar calculation, where we take a + sign in the formula for the solution of a quadratic equation, shows that $s_{0}(T)$ is a strictly increasing function of $\beta$, and so $s_{0}(\tilde{T}) \leq s_{0}(T)$ if and only if $A * A^{-T} \geq \tilde{A} * \tilde{A}^{-T}$. Thus $\left(1^{\prime}\right) \Rightarrow\left(2^{\prime}\right)$.

$(2) \Leftrightarrow(3)$. For any invertible matrix $M$, the row sums of $M * M^{-T}$ are all 1 . Hence $A * A^{-T}-\tilde{A} * \tilde{A}^{-T}$ has row sums zero, and is therefore of rank 1 . Now

$$
A * A^{-T}-\tilde{A} * \tilde{A}^{-T}=\frac{1}{\Delta}\left[\begin{array}{cc}
k_{11} k_{22} & -k_{12} k_{21} \\
-k_{21} k_{12} & k_{11} k_{22}
\end{array}\right]-\frac{1}{\tilde{\Delta}}\left[\begin{array}{cc}
\tilde{k}_{11} \tilde{k}_{22} & -\tilde{k}_{12} \tilde{k}_{21} \\
-\tilde{k}_{21} \tilde{k}_{12} & \tilde{k}_{11} \tilde{k}_{22}
\end{array}\right]
$$

a rank one Hermitian matrix with equal diagonal entries. Such a matrix is negative if and only if its $(1,1)$ entry (which is half its trace) is non-positive. Thus condition (2) is equivalent to

$$
\frac{k_{11} k_{22}}{\Delta} \leq \frac{\tilde{k}_{11} \tilde{k}_{22}}{\tilde{\Delta}}
$$

Since

$$
K=\left[\begin{array}{cc}
A & u \\
u^{*} & k_{33}
\end{array}\right], \quad u=\left[\begin{array}{l}
k_{13} \\
k_{23}
\end{array}\right]
$$

we have

$$
\operatorname{det} K=k_{33} \operatorname{det}\left(A-k_{33}^{-1} u u^{*}\right)=k_{33} \operatorname{det} \tilde{A}=k_{33} \tilde{\Delta} .
$$

Hence (3) is equivalent to

$$
k_{11} k_{22} k_{33}^{-1} \operatorname{det} K \leq \tilde{k}_{11} \tilde{k}_{22} \operatorname{det} A,
$$

that is, to

$$
k_{11} k_{22} k_{33} \operatorname{det} K \leq k_{33}^{2}\left(k_{11}-\frac{k_{13} k_{31}}{k_{33}}\right)\left(k_{22}-\frac{k_{23} k_{32}}{k_{33}}\right)\left(k_{11} k_{22}-k_{12} k_{21}\right) .
$$

This simplifies to

$$
k_{11} k_{22} k_{33} \operatorname{det} K \leq\left(k_{22} k_{33}-\left|k_{23}\right|^{2}\right)\left(k_{11} k_{33}-\left|k_{13}\right|^{2}\right)\left(k_{11} k_{22}-\left|k_{12}\right|^{2}\right) .
$$

Thus $(2) \Leftrightarrow(3)$. An easy modification of the argument shows that $\left(2^{\prime}\right) \Leftrightarrow\left(3^{\prime}\right)$. 
Note that condition (3) is symmetric with respect to permutations of $\Omega=$ $\{1,2,3\}$. From $(2) \Leftrightarrow(3)$ it follows that (2) is equivalent to the corresponding inequality obtained by choosing $\alpha$ to be 1 or 2 instead of 3 . This completes the proofs that $(2) \Rightarrow(1)$ and $\left(2^{\prime}\right) \Rightarrow\left(1^{\prime}\right)$.

$(3) \Leftrightarrow(4)$ and $\left(3^{\prime}\right) \Leftrightarrow\left(4^{\prime}\right)$ are consequences of the identity

$$
\begin{aligned}
& k_{11} k_{22} k_{33} \operatorname{det} K-\left(k_{22} k_{33}-\left|k_{23}\right|^{2}\right)\left(k_{11} k_{33}-\left|k_{13}\right|^{2}\right)\left(k_{11} k_{22}-\left|k_{12}\right|^{2}\right) \\
& =\left(\prod_{i, j=1}^{3} k_{i j}\right) \operatorname{det}(1 / K)
\end{aligned}
$$

which is a matter of straightforward verification.

The equivalence of $\left(1^{\prime \prime}\right)$ to $\left(4^{\prime \prime}\right)$ is immediate. The proof that $\left(2^{\prime \prime}\right) \Leftrightarrow\left(5^{\prime \prime}\right)$ will be given below; see the Remark following the proof of Theorem 3.9

Corollary 3.4. Let $H$ be the restriction of the Bergman space $L_{a}^{2}(\mathbb{D})$ to a threepoint subset of $\mathbb{D}$. Then $H$ has the compression property for $s_{1}$ but not for $s_{0}$.

Proof. Let $\Omega=\left\{\lambda_{1}, \lambda_{2}, \lambda_{3}\right\} \subset \mathbb{D}$ consist of three distinct points and $H=L_{a}^{2}(\mathbb{D}) \mid \Omega$. Then $H$ can be identified with the 3 -dimensional subspace $\operatorname{span}\left\{k_{\lambda_{1}}, k_{\lambda_{2}}, k_{\lambda_{3}}\right\}$ of $L_{a}^{2}(\mathbb{D})$, where $k$ is the Bergman kernel of $\mathbb{D}$,

$$
k(\lambda, \mu)=(1-\lambda \bar{\mu})^{-2} .
$$

The reproducing kernel $k_{H}$ of $H$ is the restriction of $k$ to $\Omega \times \Omega$. Thus, for $1 \leq i, j \leq$ 3 ,

$$
1 / k_{H}\left(\lambda_{i}, \lambda_{j}\right)=1-2 \lambda_{i} \bar{\lambda}_{j}+\lambda_{i}^{2} \bar{\lambda}_{j}^{2}
$$

That is,

$$
1 / K_{H}=\left[1 / k_{H}\left(\lambda_{i}, \lambda_{j}\right)\right]_{i, j=1}^{3}=V^{*} \operatorname{diag}\{1,-2,1\} V
$$

where $V$ is the non-singular Vandermonde matrix

$$
V=\left[\begin{array}{ccc}
1 & 1 & 1 \\
\lambda_{1} & \lambda_{2} & \lambda_{3} \\
\lambda_{1}^{2} & \lambda_{2}^{2} & \lambda_{3}^{2}
\end{array}\right]
$$

Thus

$$
\operatorname{det}\left(1 / K_{H}\right)=-2|\operatorname{det} V|^{2}<0 .
$$

It follows from Theorem 3.1 that $H$ has the compression property for $s_{1}$ but not for $s_{0}$.

Corollary 3.5. Let $H$ be a Hilbert space of functions on a three-point set $\Omega$ with non-singular kernel $k$, and suppose that $k$ takes the value zero at some point of $\Omega \times \Omega$. Then $H$ has the compression property for $s_{1}$. Moreover, $H$ has the compression property for $s_{0}$ if and only if $k$ vanishes at at least four points of $\Omega \times \Omega$.

Proof. Suppose $\Omega=\{1,2,3\}$ and $k_{12}=0$. By Theorem 3.1 and the note following it $H$ has the compression property for $s_{1}$ if and only if

$$
\left|\begin{array}{ccc}
0 & k_{11} k_{13} & 0 \\
k_{22} k_{23} & 0 & 0 \\
k_{32} k_{33} & k_{31} k_{33} & k_{31} k_{32}
\end{array}\right| \leq 0
$$


that is, if and only if

$$
-k_{11} k_{22}\left|k_{23} k_{13}\right|^{2} \leq 0
$$

which is true. On the other hand, the compression property for $s_{0}$ holds in $H$ if and only if the last inequality holds with equality, which is so if and only if $k_{23}=0$ or $k_{13}=0$.

On combining Theorem 3.1 with Lemma 2.1 and Corollary 3.5 we obtain the following.

Theorem 3.6. Let $H$ be a Hilbert function space with non-singular kernel $k$ on a set $\Omega$ containing at least 3 points. Suppose that $H$ has the compression property for both $s_{0}$ and $s_{1}$ for pairs of points of $\Omega$. Then for any 3-point subset $\Omega^{\prime}$ of $\Omega$, the restriction of $k$ to $\Omega^{\prime} \times \Omega^{\prime}$ is either a Szegö kernel or has at least 4 zeros on $\Omega^{\prime} \times \Omega^{\prime}$.

Example 2.3 shows that it does not follow, for non-vanishing $k$, that $k$ is itself a Szegö kernel. We therefore need to study compression properties on 4-point sets. Let $\Omega=\{1,2,3,4\}$, let $k$ be a positive non-singular kernel on $\Omega$ and let $H$ be the corresponding Hilbert space of functions on $\Omega$. We shall analyze the extension of functions from $\{1,2,3\}$ to $\Omega$ with preservation of $s_{1}$. For data $z_{1}, z_{2}, z_{3} \in \mathbb{C}$ let $T, \tilde{T}$ be the operators of Definition 1.5; we need to find the conditions on $k$ that ensure $s_{1}(\tilde{T}) \leq s_{1}(T)$ whatever the $z_{j}$. By Lemma 3.3 the characteristic polynomial of $T^{*} T$ acting on $\operatorname{span}\left\{k_{1}, k_{2}, k_{3}\right\}$ is

$$
p(t) \stackrel{\text { def }}{=} \Delta^{-1} \operatorname{det}\left[\left(t-z_{i} \bar{z}_{j}\right) k_{i j}\right]_{i, j=1}^{3}
$$

where $\Delta=\operatorname{det}\left[k_{i j}\right]_{i, j=1}^{3}$. Regard the $j$ th column of the matrix $\left[\left(t-z_{i} \bar{z}_{j}\right) k_{i j}\right]$ as the sum of the two columns $\left[t k_{i j}\right]_{i=1}^{3}$ and $\left[z_{i} \bar{z}_{j} k_{i j}\right]_{i=1}^{3}$, and expand the right hand side accordingly as the sum of eight determinants, of which one, for example, is

$$
\left|\begin{array}{ccc}
z_{1} \bar{z}_{1} k_{11} & t k_{12} & z_{1} \bar{z}_{3} k_{13} \\
z_{2} \bar{z}_{1} k_{21} & t k_{22} & z_{2} \bar{z}_{3} k_{23} \\
z_{3} \bar{z}_{1} k_{31} & t k_{32} & z_{3} \bar{z}_{3} k_{33}
\end{array}\right| .
$$

On expanding further we find that the coefficient of $t$ in $p(t)$ is

$$
\Delta^{-1} \sum_{i, j=1}^{3} k_{i j} C_{i j} Z_{i} \bar{Z}_{j}
$$

where $C_{i j}$ is the $(i, j)$ cofactor of $C=\left[k_{i j}\right]_{i, j=1}^{3}$ and $Z^{T}=\left[\begin{array}{lll}z_{2} z_{3} & z_{3} z_{1} & z_{1} z_{2}\end{array}\right]$. Since $\Delta^{-1} C_{i j}$ is the $(j, i)$ entry of $C^{-1}$ we can write this in the form

$$
\left\langle C * C^{-T} Z, Z\right\rangle_{\mathbb{C}^{3}} .
$$

Likewise the coefficient of $t^{2}$ is

$$
-\Delta^{-1} \sum_{i j} k_{i, j} C_{i j} z_{i} \bar{z}_{j}=\left\langle C * C^{-T} z, z\right\rangle_{\mathbb{C}^{3}}
$$

where $z^{T}=\left[\begin{array}{lll}z_{1} & z_{2} & z_{3}\end{array}\right]$. Let us introduce the following notation for a $3 \times 3$ matrix $A>0$ :

$$
p_{A}(t) \stackrel{\text { def }}{=} t^{3}-\left\langle A * A^{-T} z, z\right\rangle t^{2}+\left\langle A * A^{-T} Z, Z\right\rangle t-\left|z_{1} z_{2} z_{3}\right|^{2}
$$

where $z^{T}=\left[\begin{array}{lll}z_{1} & z_{2} & z_{3}\end{array}\right], Z^{T}=\left[\begin{array}{lll}z_{2} z_{3} & z_{3} z_{1} & z_{1} z_{2}\end{array}\right]$. Since the characteristic polynomials of $T^{*} T$ and $\tilde{T}^{*} \tilde{T}$ are monic and have constant terms equal to $-|\operatorname{det} T|^{2}$ $=-\left|z_{1} z_{2} z_{3}\right|^{2}$, we obtain the following. 
Lemma 3.7. Let $T, \tilde{T}$ be as in Definition 1.5 for the points $1,2,3$ and $\alpha=4$. The characteristic polynomials of $T^{*} T$ and $\tilde{T}^{*} \tilde{T}$ are $p_{C}$ and $p_{\tilde{C}}$ respectively, where

$$
C=\left[k_{i j}\right]_{i, j=1}^{3}, \quad \tilde{C}=\left[k_{i j}-\frac{k_{i 4} k_{4 j}}{k_{44}}\right]_{i, j=1}^{3} .
$$

To say that $s_{1}(\tilde{T}) \leq s_{1}(T)$ is thus to say that the second zero of $p_{\tilde{C}}$ is less than or equal to the second zero of $p_{C}$.

Lemma 3.8. Let $A, B$ be positive definite $3 \times 3$ matrices such that, for any choice of $z_{1}, z_{2}, z_{3} \in \mathbb{C}$, the second zero of $p_{A}$ is no greater than the second zero of $p_{B}$. Then

$$
A * A^{-T}=B * B^{-T} .
$$

Proof. Write $A * A^{-T}=\left[a_{i j}\right], \quad B * B^{-T}=\left[b_{i j}\right]$. Take $z_{3}=0$. Then

$$
p_{A}(t)=t\left(t^{2}-\left\langle A * A^{-T} z, z\right\rangle t+a_{33}\left|z_{1} z_{2}\right|^{2}\right) .
$$

Since all zeros of $p_{A}$ are non-negative,

$$
\left\langle A * A^{-T} z, z\right\rangle^{2} \geq 4 a_{33}\left|z_{1} z_{2}\right|^{2},
$$

and moreover the second zero of $p_{A}$ is the lesser zero of the quadratic factor on the right hand side of (3.3). We shall use the following elementary observation. Suppose $\beta_{j}, \gamma_{j}$ are positive real numbers for $j=1,2$ such that $\beta_{j}^{2} \geq 4 \gamma_{j}$, and let $q_{j}(t)=t^{2}-\beta_{j} t+\gamma_{j}$. Then the lesser zero of $q_{1}$ is no greater than that of $q_{2}$ if and only if

$$
\gamma_{1}\left(\beta_{2}+\sqrt{\beta_{2}^{2}-4 \gamma_{2}}\right) \leq \gamma_{2}\left(\beta_{1}+\sqrt{\beta_{1}^{2}-4 \gamma_{1}}\right) .
$$

Apply this observation with $p_{A}=t q_{1}, p_{B}=t q_{2}$ (so that $\beta_{1}=\left\langle A * A^{-T} z, z\right\rangle$, $\gamma_{1}=a_{33}\left|z_{1} z_{2}\right|^{2}$, etc.). We deduce that, for all $z_{1}, z_{2} \in \mathbb{C}$,

$$
\begin{aligned}
& a_{33}\left|z_{1} z_{2}\right|^{2}\left(\left\langle B * B^{-T} z, z\right\rangle+\sqrt{\left\langle B * B^{-T} z, z\right\rangle^{2}-4 b_{33}\left|z_{1} z_{2}\right|^{2}}\right) \\
\leq & b_{33}\left|z_{1} z_{2}\right|^{2}\left(\left\langle A * A^{-T} z, z\right\rangle+\sqrt{\left\langle A * A^{-T} z, z\right\rangle^{2}-4 a_{33}\left|z_{1} z_{2}\right|^{2}}\right) .
\end{aligned}
$$

Divide through by $\left|z_{1} z_{2}\right|^{2}$ and then put $z_{1}=1, z_{2}=0$ to obtain

$$
a_{33} b_{11} \leq b_{33} a_{11} \text {. }
$$

By symmetry the opposite inequality also holds, and thus

$$
a_{i i} b_{j j}=b_{i i} a_{j j}, \quad 1 \leq i, j \leq 3 .
$$

Hence there exists $\tau>0$ such that $a_{i i}=\tau b_{i i}, 1 \leq i \leq 3$. Cancel $\left|z_{1} z_{2}\right|^{2}$ from (3.4) and put $z_{2}=\xi \in \mathbb{R}$ to get

$$
\begin{aligned}
& a_{33}\left(\left\langle B * B^{-T} z, z\right\rangle+\sqrt{\left\langle B * B^{-T} z, z\right\rangle^{2}-4 b_{33}\left|z_{1}\right|^{2} \xi^{2}}\right) \\
\leq & b_{33}\left(\left\langle A * A^{-T} z, z\right\rangle+\sqrt{\left\langle A * A^{-T} z, z\right\rangle^{2}-4 a_{33}\left|z_{1}\right|^{2} \xi^{2}}\right)
\end{aligned}
$$


for all $z_{1} \in \mathbb{C}, \xi \in \mathbb{R}$, where $z^{T}=\left[\begin{array}{lll}z_{1} & \xi & 0\end{array}\right]$. The two sides of this inequality are equal when $\xi=0$, and so the inequality remains true if we differentiate and put $\xi=0$. Now

$$
\begin{aligned}
\left.\frac{d}{d \xi} \sqrt{\left\langle B * B^{-T} z, z\right\rangle^{2}-4 \xi^{2}\left|z_{1}\right|^{2} b_{33}}\right|_{\xi=0} & =\left.\frac{d}{d \xi}\left\langle B * B^{-T} z, z\right\rangle\right|_{\xi=0} \\
& =2 \operatorname{Re}\left(b_{21} z_{1}\right) .
\end{aligned}
$$

Hence

$$
a_{33} 2 \operatorname{Re}\left(b_{21} z_{1}\right) \leq b_{33} 2 \operatorname{Re}\left(a_{21} z_{1}\right)
$$

Thus, for all $z_{1} \in \mathbb{C}$,

$$
\operatorname{Re}\left\{z_{1}\left(a_{33} b_{21}-b_{33} a_{21}\right)\right\} \leq 0
$$

and hence

$$
a_{33} b_{21}-b_{33} a_{21}=0 .
$$

Since $a_{33}=\tau b_{33}$, we have $a_{21}=\tau b_{21}$. By symmetry $a_{i j}=\tau b_{i j}$ for each $i, j$, and so

$$
A * A^{-T}=\tau B * B^{-T} .
$$

Since the row sums of $A * A^{-T}$ and $B * B^{-T}$ are all 1 , we must have $\tau=1$, and so

$$
A * A^{-T}=B * B^{-T} \text {. }
$$

We shall say that two $n \times n$ matrices $A, B$ are diagonally congruent if there is a non-singular diagonal matrix $D$ such that $A=D^{*} B D$. In the event that the entries of $A$ and $B$ are all non-zero it is equivalent to saying that the (entrywise) ratio $A / B$ has rank 1 .

Theorem 3.9. Let $H$ be a Hilbert space of functions with a non-vanishing and non-singular kernel $k$ on the 4-point set $\Omega=\{1,2,3,4\}$. The following conditions are equivalent.

(1) $H$ has the compression property for $s_{1}$ for triples of points;

(2) if

$$
A=\left[k_{i j}\right]_{i, j=1}^{3}, \quad \tilde{A}=\left[k_{i j}-\frac{k_{i 4} k_{4 j}}{k_{44}}\right]_{i, j=1}^{3},
$$

then $A * A^{-T}=\tilde{A} * \tilde{A}^{-T}$

(3) $K^{-T}$ is diagonally congruent to $K$, where $K=\left[k_{i j}\right]_{i, j=1}^{4}$.

Proof. (1) $\Rightarrow(2)$ In the definition of compression property (Definition 1.5) take $\lambda_{1}=1, \lambda_{2}=2, \lambda_{3}=3$ and $\alpha=4$. For any choice of data $z_{1}, z_{2}, z_{3} \in \mathbb{C}$ we have $s_{1}(\tilde{T}) \leq s_{1}(T)$. By Lemma 3.7 it follows that the second zero of $p_{\tilde{A}}$ does not exceed that of $p_{A}$, and so, by Lemma 3.8, (2) holds.

$(2) \Leftrightarrow(3)$ We shall show that both are equivalent to a fourth condition involving

in fact to

$$
c \stackrel{\text { def }}{=} k_{44}, \quad u \stackrel{\text { def }}{=}\left[\begin{array}{lll}
k_{14} & k_{24} & k_{34}
\end{array}\right]^{T},
$$

(4) there exists a non-singular diagonal matrix $P$ such that $A^{-T}=P \tilde{A} P^{*}$ and

$$
A P^{*} \bar{u}=\left(\frac{c}{c-u^{*} A^{-1} u}\right)^{\frac{1}{2}} u .
$$


Note that $\tilde{A}=A-c^{-1} u u^{*}$ and that $c-u^{*} A^{-1} u$, being the Schur complement of $A$ in $K$, is positive. Moreover one may verify that $\tilde{A}^{-1}=A^{-1}+w w^{*}$ where

$$
w=\frac{A^{-1} u}{\left(c-u^{*} A^{-1} u\right)^{\frac{1}{2}}} .
$$

Hence

$$
\begin{aligned}
\tilde{A} * \tilde{A}^{-T} & =\tilde{A} *\left(A^{-T}+\bar{w} w^{T}\right) \\
& =\left(A-c^{-1} u u^{*}\right) * A^{-T}+\tilde{A} * \bar{w} w^{T},
\end{aligned}
$$

and so (2) is equivalent to

$$
c^{-1} u u^{*} * A^{-T}=\bar{w} w^{T} * \tilde{A}
$$

Suppose (2) holds. The components of $u$ are non-zero by hypothesis, and hence those of $w$ are too. Let $P=\operatorname{diag}\left\{c^{\frac{1}{2}} \bar{w}_{j} / u_{j}\right\}$; then (1.5) gives $A^{-T}=P \tilde{A} P^{*}$, while $P^{*} \bar{u}=c^{\frac{1}{2}} w$, and so

$$
A P^{*} \bar{u}=c^{\frac{1}{2}} A w=\left(\frac{c}{c-u^{*} A^{-1} u}\right)^{\frac{1}{2}} u .
$$

Thus $(2) \Rightarrow(4)$. Conversely, if (4) holds, then $P^{*} \bar{u}=c^{\frac{1}{2}} w$ and the relation $A^{-T}=$ $P \tilde{A} P^{*}$ yields (3.6). Thus $(4) \Rightarrow(2)$.

$(3) \Leftrightarrow(4)$ Note that

$$
K=\left[\begin{array}{cc}
A & u \\
u^{*} & c
\end{array}\right]=\left[\begin{array}{cc}
1 & c^{-1} u \\
0 & 1
\end{array}\right]\left[\begin{array}{cc}
\tilde{A} & 0 \\
0 & c
\end{array}\right]\left[\begin{array}{cc}
1 & 0 \\
c^{-1} u^{*} & 1
\end{array}\right]
$$

so that

$$
\begin{aligned}
K^{-T} & =\left[\begin{array}{cc}
1 & 0 \\
-c^{-1} u^{T} & 1
\end{array}\right]\left[\begin{array}{cc}
\tilde{A}^{-T} & 0 \\
0 & c^{-1}
\end{array}\right]\left[\begin{array}{cc}
1 & -c^{-1} \bar{u} \\
0 & 1
\end{array}\right] \\
& =\left[\begin{array}{cc}
\tilde{A}^{-T} & -c^{-1} \tilde{A}^{-T} \bar{u} \\
-c^{-1} u^{T} \tilde{A}^{-T} & c^{-1}+c^{-2} u^{T} \tilde{A}^{-T} \bar{u}
\end{array}\right] .
\end{aligned}
$$

Suppose (4) holds. Then $\tilde{A}^{-T}=P A P^{*}$ and so

$$
\begin{aligned}
K^{-T} & =\left[\begin{array}{cc}
P A P^{*} & -c^{-1} P A P^{*} \bar{u} \\
-c^{-1} u^{T} P A P^{*} & c^{-1}+c^{-2} u^{T} P A P^{*} \bar{u}
\end{array}\right] \\
& =\left[\begin{array}{cc}
P A P^{*} & -c^{-1} \kappa P A u \\
-c^{-1} \kappa U^{*} A P^{*} & c^{-1}+c^{-2} u^{T} P A P^{*} \bar{u}
\end{array}\right]
\end{aligned}
$$


where $\kappa=c^{\frac{1}{2}}\left(c-u^{*} A^{-1} u\right)^{-\frac{1}{2}}$. Now

$$
\begin{aligned}
c^{-1}+c^{-2} u^{T} P A P^{*} \bar{u} & =c^{-1}+c^{-2} \kappa^{2} u^{*} A^{-1} u \\
& =c^{-1}+c^{-1}\left(c-u^{*} A^{-1} u\right)^{-1} u^{*} A^{-1} u \\
& =\left(c-u^{*} A^{-1} u\right)^{-1}=c^{-1} \kappa^{2}
\end{aligned}
$$

It follows that

$$
\begin{aligned}
K^{-T} & =\left[\begin{array}{cc}
P A P^{*} & -c^{-1} \kappa P u \\
-c^{-1} \kappa u^{*} P^{*} & c^{-1} \kappa^{2}
\end{array}\right] \\
& =\left[\begin{array}{cc}
P & 0 \\
0 & -c^{-1} \kappa
\end{array}\right]\left[\begin{array}{cc}
A & u \\
u^{*} & c
\end{array}\right]\left[\begin{array}{cc}
P^{*} & 0 \\
0 & -c^{-1} \kappa
\end{array}\right] \\
& =\Lambda K \Lambda^{*}
\end{aligned}
$$

where $\Lambda$ is the non-singular diagonal matrix $\operatorname{diag}\left\{P,-c^{-1} \kappa\right\}$. Thus $(4) \Rightarrow(3)$.

Suppose (3) holds: say $K^{-T}=\Lambda K \Lambda^{*}$ where $\Lambda$ is a non-singular diagonal matrix which we write as $\operatorname{diag}\{Q, \gamma\}$ for some non-singular diagonal $3 \times 3$ matrix $Q$ and $\gamma \neq 0$. By virtue of (3) we have

$$
\begin{gathered}
\tilde{A}^{-T}=Q A Q^{*}, \\
-c^{-1} \tilde{A}^{-T} \bar{u}=\bar{\gamma} Q u, \\
c^{-1}+c^{-2} u^{T} \tilde{A}^{-T} \bar{u}=c|\gamma|^{2} .
\end{gathered}
$$

From the first two equations we have

$$
Q A Q^{*} \bar{u}=-c \bar{\gamma} Q u
$$

and hence

$$
A Q^{*} \bar{u}=-c \bar{\gamma} u
$$

and moreover

$$
\begin{aligned}
c^{-2} u^{T} \tilde{A}^{-T} \bar{u} & =c^{-2} u^{T} \tilde{A}^{-T} \tilde{A}^{T} \tilde{A}^{-T} \bar{u} \\
& =\gamma u^{*} Q^{*} \tilde{A}^{T} \bar{\gamma} Q u \\
& =|\gamma|^{2} u^{*} Q^{*}\left(Q^{-*} A^{-1} Q^{-1}\right) Q u \\
& =|\gamma|^{2} u^{*} A^{-1} u,
\end{aligned}
$$

which combines with the third equation to yield

$$
c^{-1}+|\gamma|^{2} u^{*} A^{-1} u=c|\gamma|^{2}
$$

and hence

$$
|\gamma|^{2}=c^{-1}\left(c-u^{*} A^{-1} u\right)^{-1} .
$$

It follows that there is a complex number $\xi$ of unit modulus such that

$$
\xi \gamma=-|\gamma|=-c^{-\frac{1}{2}}\left(c-u^{*} A^{-1} u\right)^{-\frac{1}{2}}
$$

Let $P=\xi Q$. Then equations (3.7), (3.8) become

$$
\begin{aligned}
\tilde{A}^{-T} & =P A P^{*}, \\
A P^{*} \bar{u} & =c|\gamma| u \\
& =c^{\frac{1}{2}}\left(c-u^{*} A^{-1} u\right)^{-\frac{1}{2}} u .
\end{aligned}
$$

Thus $(3) \Rightarrow(4)$. 
It remains to prove that $(2) \Rightarrow(1)$. Suppose, then, that $A * A^{-T}=\tilde{A} * \tilde{A}^{-T}$. Then $p_{A}=p_{\tilde{A}}$, so that $T, \tilde{T}$ (as defined at the beginning of the proof) have the same singular values, and in particular $s_{1}(\tilde{T}) \leq s_{1}(T)$. However, to prove (1) one must show that the corresponding relation holds for any triple of points from $\Omega$, not just $\{1,2,3\}$. This conclusion follows from the equivalence of (2) and (3) and the symmetry of condition (3).

Remarks. 1. The proof of $(2) \Leftrightarrow(3)$ remains valid for $n \times n$ positive matrices $K$ with trivial modifications. The case $n=3$ is $\left(2^{\prime}\right) \Leftrightarrow\left(5^{\prime}\right)$ in Lemma 3.2.

2. One way that (2) can hold is if $\tilde{A}$ is diagonally congruent to $A$, and this is in fact another characterization of Szegő kernels. One might suspect that the conditions of the theorem are equivalent to $k$ being a Szegö kernel, but this is false.

Example 3.10. There are non-vanishing positive non-singular kernels on $\{1,2,3,4\}$ which have the compression property for $s_{1}$ for triples of points but not for pairs of points. Such kernels of course cannot be Szegö kernels. To see this consider positive definite $2 \times 2$ matrices $M, N$ with non-zero entries. Up to diagonal congruence they have the form

$$
M=\left[\begin{array}{cc}
1 & 1 \\
1 & m
\end{array}\right], \quad N=\left[\begin{array}{ll}
1 & 1 \\
1 & n
\end{array}\right]
$$

where $m, n>1$. It is easy to see that $M, N$ are diagonally congruent to their inverse transposes. This property is clearly preserved under tensor products, and so the $4 \times 4$ matrix $M \otimes N$ has the properties (1) to (3) of Theorem 3.9. However, the leading principal $3 \times 3$ submatrix of $M \otimes N$ is

$$
\left[\begin{array}{ccc}
1 & 1 & 1 \\
1 & m & 1 \\
1 & 1 & n
\end{array}\right]
$$

and the determinant of the reciprocal of this matrix is $\left(m^{-1}-1\right)\left(n^{-1}-1\right)$, which is positive. By Theorem 3.1, $M \otimes N$ does not have the compression property for $s_{1}$ for pairs of points.

With the aid of Theorem 3.9 and a matrix algebra programme it is easy to show that the Bergman space $L_{a}^{2}(\mathbb{D})$ does not have the compression property for $s_{1}$, despite the fact that its 3 -point restrictions do. Choose 4 points in $\mathbb{D}\left(0, \pm \frac{1}{2}, \frac{3}{4}\right.$ will do), and form the corresponding matrix $K=\left[\left(1-\lambda_{i} \bar{\lambda}_{j}\right)^{-2}\right]_{i, j=1}^{4}$. A couple of commands will produce the singular values of the matrix $K^{-T} / K$. They turn out to be all non-zero, so $K^{-T} / K$ is not rank 1 , or in other words, $K^{-T}$ is not diagonally congruent to $K$. Thus $L_{a}^{2}(\mathbb{D})$ does not have the compression property for $s_{1}$ for triples of points.

We now show that the assumption of quite modest compression properties forces a kernel to be Szegö.

Theorem 3.11. Let $H$ be a Hilbert space of functions with non-singular kernel $k$ on a set $\Omega$ containing at least 4 points. Suppose that $k$ does not vanish on $\Omega \times \Omega$ and that

(1) $H$ has the compression property for $s_{0}$ and $s_{1}$ for pairs of points, and

(2) $H$ has the compression property for $s_{1}$ for triples of points.

Then $k$ is a Szegö kernel. 
Proof. In view of Lemma 2.2 we can assume that $\Omega=\{1,2,3,4\}$. Write

$$
K=\left[k_{i j}\right]_{i, j=1}^{4}=\left[\begin{array}{cc}
A & u \\
u^{*} & c
\end{array}\right] .
$$

By assumption (1) and Lemma 3.2 there is a non-singular diagonal matrix $P$ such that $A^{-T}=P A P^{*}$. Another way of saying this is: there is a column vector $p \in \mathbb{C}^{3}$ with non-zero components such that

$$
A^{-T}=p p^{*} * A
$$

By assumption (2) and Theorem 3.9 we have also

$$
A * A^{-T}=\tilde{A} * \tilde{A}^{-T}
$$

where $\tilde{A}=A-c^{-1} u u^{*}$. Since $\tilde{A}^{-1}=A^{-1}+w w^{*}$ for a suitable column matrix $w$ we have

$$
A * A^{-T}=\left(A-c^{-1} u u^{*}\right) *\left(A^{-T}+\bar{w} w^{T}\right)
$$

and hence

$$
\begin{aligned}
0 & =A * \bar{w} w^{T}-c^{-1} u u^{*} * A^{-T}-c^{-1} u u^{*} * \bar{w} w^{T} \\
& =A *\left(\bar{w} w^{T}-c^{-1} u u^{*} * p p^{*}\right)-c^{-1} u u^{*} * \bar{w} w^{T}
\end{aligned}
$$

Thus

$$
A=\frac{c^{-1} u u^{*} * \bar{w} w^{T}}{\bar{w} w^{T}-c^{-1} u u^{*} * p p^{*}}
$$

(note that our hypotheses force all components of $u$ and $w$ to be non-zero, and hence those of the denominator are non-zero too). Consequently

$$
\begin{aligned}
1 / A & =\frac{c \bar{w} w^{T}-u u^{*} * p p^{*}}{u u^{*} * \bar{w} w^{T}} \\
& =\frac{c}{u u^{*}}-\frac{p p^{*}}{\bar{w} w^{T}} .
\end{aligned}
$$

Hence

$$
\begin{aligned}
1 / K & =\left[\begin{array}{cc}
1 / A & 1 / u \\
1 / u^{*} & 1 / c
\end{array}\right] \\
& =\left[\begin{array}{cc}
\frac{c}{u u^{*}}-\frac{p p^{*}}{\bar{w} w^{T}} & \frac{1}{u} \\
\frac{1}{u^{*}} & \frac{1}{c}
\end{array}\right] .
\end{aligned}
$$

On multiplying the second block row by $-c / u$ and adding to the first block row we infer that $1 / K$ is row-equivalent to

$$
\left[\begin{array}{cc}
-\frac{p p^{*}}{\bar{w} w^{T}} & 0 \\
\frac{1}{u^{*}} & \frac{1}{c}
\end{array}\right]
$$

which has rank 2. Thus $1 / K$ has rank 2, which is to say that $k$ is Szegö. 
Theorem 3.12. Let $H$ be a Hilbert space of functions with non-singular kernel $k$ on a set $\Omega$ containing at least 4 points. Suppose that $k$ does not vanish on $\Omega \times \Omega$ and that every cofinite set of uniqueness for $H$ in $\Omega$ is also a set of uniqueness for $H H$. If $H$ has the $0-A A K$ property and the 1-AAK property, then $k$ is a Szegö kernel.

Proof. It follows from Theorem 1.8 that $H$ has the compression property for $s_{0}$ and $s_{1}$. By Theorem $3.11, k$ is a Szegö kernel.

We leave open the following question: Is there a significant class of Hilbert function spaces which have the 1-AAK property but not the 0-AAK property?

\section{REFERENCES}

[AAK] V. M. Adamyan, D. Z. Arov and M. G. Krein, Analytic properties of Schmidt pairs of a Hankel operator and generalized Schur-Takagi problem, Mat. Sbornik 86 (1971), 33-73. MR 45:7505

[Ag1] J. Agler, Interpolation, preprint (1987).

[Ag2] J. Agler, Nevanlinna-Pick interpolation on Sobolev space, Proc. Amer. Math. Soc. 108(1990) 341-351. MR 90f:30041

[AY] J. Agler and N. J. Young, Functions which are almost multipliers of Hilbert function spaces, to appear in Proc. London Math. Soc. CMP 98:06

[Ak] N. I. Akhiezer, On a minimum problem in the theory of functions and on the number of roots of an algebraic equation which lie inside the unit circle, Izv. Akad. Nauk SSSR 9(1931) 1169-1189.

[Ar] N. Aronszajn, Theory of reproducing kernels, Trans. Amer. Math. Soc. 68 (1950), 337404. MR 14:479c

[BH] J. A. Ball and J. W. Helton, A Beurling-Lax theorem for the Lie group $U(m, n)$ which contains most classical interpolation theory, J. Operator Theory 9(1983) 107-142. MR 84m: 47046

[CG] T. Constantinescu and A. Gheondea, Minimal signature in lifting of operators I, $J$. Operator Theory 22 (1989) 345-367. MR 91b:47013

[CS] M. Cotlar and C. Sadosky, Nehari and Nevalinna-Pick problems and holomorphic extensions in the polydisk in terms of restricted BMO, J. Functional Analysis 124 (1994) 205-210. MR 95f:47047

[DFT] J. C. Doyle, B. A. Francis and A. R. Tannenbaum, Feedback Control Theory, MaxwellMacmillan Publishing Co., New York 1992. MR 93k:93002

[DGK] P. Delsarte, Y. Genin and Y. Kamp, On the role of the Nevanlinna-Pick problem in circuit and system theory, Circuit Theory and Applications 9 (1981) 177-187. MR 82d:94052

[FF] C. Foias and A. E. Frazho, The Commutant Lifting Approach to Interpolation Problems, OT44, Birkhäuser Verlag, Basel 1986. MR 92k:47033

[G] K. Glover, All optimal Hankel-norm approximations of linear multivariable systems and their $L^{\infty}$ error bounds, Int. J. Control 39(1984) 1115-1193. MR 86a:93029

[GRSW] I. Gohberg, L. Rodman, T. Shalom and H. J. Woerdemann, Bounds for eigenvalues and singular values of matrix completions, Linear and Multilinear Algebra 33 (1993) 233-249. MR 96h:15019

$[\mathrm{H}] \mathrm{J}$. W. Helton, Operator theory, analytic functions, matrices and electrical engineering, CBMS Regional Conference Series No. 68, AMS, Providence 1987. MR 89f: 47001

[K] I. Kaplansky, Linear Algebra and Geometry, Chelsea Publishing Co., New York, 1969. MR 40:2689

[MS] D. E. Marshall and C. Sundberg, Interpolating sequences for multipliers of the Dirichlet space, to appear.

[P] G. Pick, Über die Beschränkungen analytischer Funktionen, welche durch vorgegebene Funktionswerte bewirkt werden, Math. Ann. 77 (1916), 7-23.

[Q1] P. Quiggin, For which reproducing kernel Hilbert spaces is Pick's theorem true? Integral Equations and Operator Theory 16 (1993), 244-266. MR 94a:47026 
[Q2] P. Quiggin, Generalisations of Pick's Theorem to Reproducing Kernel Hilbert Spaces, Ph.D. thesis, Lancaster University, 1994.

[S] D. Sarason, Generalized interpolation in $H^{\infty}$, Trans. Amer. Math. Soc. 127 (1967) 179-203. MR 34:8193

Department of Mathematics, University of California at San Diego, La Jolla, CalIFORNIA 92093

E-mail address: jagler@ucsd.edu

Department of Mathematics, University of Newcastle, Newcastle upon Tyne Ne1 7RU, ENGLAND

E-mail address: N.J.Young@ncl.ac.uk 